\title{
Strahlenbiologische Untersuchungen an Gammariden (Crustacea, Amphipoda)
}

\author{
M. HOPPENHEIT \\ Biologische Anstalt Helgoland, Zentrale, Hamburg 50
}

\begin{abstract}
Radiobiological investigations on gammarids (Crustacea, Amphipoda). Effects of single exposures of X-radiation on survival were studied in Gammarus duebeni, G. salinus and $G$. zaddachi under constant conditions of temperature $\left(15^{\circ} \mathrm{C}\right)$ and salinity $(10 \%)$. Effects on moulting were investigated in $G$. duebeni. The amphipods were irradiated with doses between 625 and 20,000 R. G. salinus and G. zaddachi are equally radioresistant $\left(\mathrm{LD}_{50 / 30}: 1,700 \mathrm{R}\right.$ ) but less resistant than $\mathrm{G}$. duebeni (LD50/30(males) : 3,900 R; LD50/30(females) : 3,500 R). At high doses, half of the test individuals die within a few days; at medium doses survival time is dose-independent; at lower doses survival time again increases with decreasing doses up to dose-ranges (below $1,250 \mathrm{R}$ for $G$. duebeni, 1,000 $\mathrm{R}$ for G. salinus and G. zaddachi), at which only a few individuals die before their natural death. Subadult gammarids are less resistant; G. duebeni of 4 to $7 \mathrm{~mm}$ body length have a $\mathrm{LD}_{50 / 30}$ of 2,200 R. Death distribution after medium doses indicates that at least two mechanisms are involved in acute mortality. A first mortality maximum occurs shortly after irradiation; subsequently, mortality decreases for a few days, and is followed be another maximum. At lower doses no individual dies during the first critical period. Shortening of survival time at higher doses results from survival of fewer individuals to the second critical period. Few or no moults occur in $G$. duebeni irradiated with $20,000 \mathrm{R}$ following the second day after irradiation and after the 30 th day in those individuals irradiated with 2,500 to $10,000 \mathrm{R}$; moulting is delayed after exposure to 5,000 to $10,000 \mathrm{R}$. Individuals of $G$. duebeni taken from the field during the mild winter 1966/67 were more resistant to radiation, and moulting was less affected, than in laboratory-reared amphipods, or in those collected in the field during other seasons. Besides on irradiation dose, survival time of an individual depends on the time of its moulting in the course of an experiment.
\end{abstract}

\section{EINLEITUNG}

Die steigende Zahl von Kernkraftwerken, die Verwendung von Kernreaktoren als Schiffsantriebe sowie die Herstellung und Erprobung von Kernwafien haben in allen an der Nutzung der Kernenergie beteiligten Ländern Untersuchungen über Probleme der Beseitigung des radioaktiven Abfalls ausgelöst. Raummangel und Sicherheitserwägungen haben bei der Suche nach geeigneten "Abfallgruben" den Blick vom Land aufs Meer gelenkt. Eine Abgabe radioaktiver Stoffe ins Meer kann aber nur dann erfolgen, wenn sichergestellt ist, daß die dem Verzehr zugeführten Meeresprodukte nicht zu einer Gefahrenquelle für den Menschen werden, und darüber hinaus die wirtschaftliche Nutzung der Meere keine Einschränkungen erleidet. Die Biologische 
Anstalt Helgoland beteiligt sich durch experimentelle Untersuchungen im Laboratorium an der Erarbeitung der Grundlagen, die für eine Beurteilung der Schädlichkeit der ionisierenden Strahlung für Meeresorganismen benötigt werden. Derartige Experimente sind zunächst an drei euryhalinen Gammariden-Arten, Gammarus duebeni, Gammarus zaddachi und Gammarus salinus, begonnen worden, deren Ergebnisse in der vorliegenden Arbeit mitgeteilt werden. Die Untersuchungen wurden in der Zeit von 1965 bis 1967 durchgeführt.

Zahlreiche Veröffentlichungen sind bereits erschienen, die über den Einfluß ionisierender Strahlung auf Krebse berichten. Häufig wurde die Strahlung nur als Hilfsmittel benutzt, um Mutationen auszulösen (GAJEWSKaja 1923) oder durch Kastration Einblick in die Steuerung der Geschlechtstätigkeit zu bekommen (HaEmmerLi-Boveri 1926, Le Roux 1933, Mori 1933a, b, Patane \& De Luca 1956, Balesdent 1965). Snider $8 x$ KerSTEN $(1935,1936)$ waren die ersten, die Krebse mit der Absicht bestrahlten, etwas über ihre Strahlenempfindlichkeit zu erfahren. BonHaM \& PaLumbo (1951) machten einige Angaben über Letaldosen bei den Amphipoden Calliopius laeviusculus und Allorchestes angustus sowie bei Eiern von Artemia salina. Uber weitere Experimente an Artemia salina berichteten Grosch \& Erdman (1955) und Grosch \& Suldrvan (1955). MORGan (1960) teilte die Ergebnisse einer strahlenbiologischen Untersuchung am Hummer mit. Hutchinson \& Easter (Hutchinson \& Easter 1960, Easter \& Hutchrnson 1961) bestrahlten Eier von Artemia salina mit $\gamma$-Strahlen, Elektronen und verschiedenen Ionen. Grosch (1962) veröffentlichte die Ergebnisse langjähriger Beobachtungen von Populationen von Artemia salina, die er in mit ${ }^{32} \mathrm{P}$ und ${ }^{65} \mathrm{Zn}$ versetztem Seewasser hielt oder mit Röntgenstrahlen bestrahlte. ReEs (1962) untersuchte die Wirkung von $\gamma$-Strahlen auf Palaemonetes pugio und Uca pugnax. Rrce (1965) berichtete über einen Versuch mit einer Laboratoriumspopulation von Tigriopus californicus in Seewasser mit ${ }^{137}$ Cs. Ballardin \& Metalli (Metalli \& Ballardin 1962, 1963, BALLARDiN \& METALLI 1965a, b) arbeiteten über die Strahlenempfindlichkeit verschiedener Stämme von Artemia salina und über den Zusammenhang von Polyploidiegrad und Auftreten von Letalmutationen. Genetische Untersuchungen am gleichen Objekt führte BowEN (1963) durch. Weitere Autoren haben sich mit der Strahlenbiologie von Artemia salina befaßt, so Cervini \& Gravelin (1965), die die Strahlenempfindlichkeit verschiedener Stadien der Oogenese feststellten. Engel \& Davis (1965) zogen Nauplien in Seewasser auf, das mit ${ }^{187} \mathrm{Cs},{ }^{60} \mathrm{Co}$ und ${ }^{32} \mathrm{P}$ versetzt war, und untersuchten das Wachstum der Tiere. Über den Einfluß des Wassergehaltes auf die Strahlenempfindlichkeit von Artemia-Eiern arbeiteren Iwasaki $(1958,1959)$ und Engel \& Fluke (1962). IWASAKI $(1965,1966)$ untersuchte ferner den Zusammenhang zwischen Strahlenschädigung und dem Auftreten von strahleninduzierten freien Radikalen. VrtaGLIANO-TADINI \& De CAPOA (1962) stellten die $\mathrm{LD}_{50}$ und $\mathrm{LD}_{100}$ für verschiedene Embryonalstadien der Wasserassel Asellus aquaticus fest. De LuCA $(1962 \mathrm{a}, \mathrm{b})$ untersuchte die Schädigung der Zellstrukturen der Mitteldarmdrüse bei der Landassel Porcellio laevis. Telitchenko (1958) hielt Daphnia magna während mehrerer Generationen in mit ${ }^{288} \mathrm{U}$, ${ }^{232} \mathrm{Th}$ und ${ }^{90+89} \mathrm{Sr}$ versetztem Wasser. Marshall $(1962,1963,1966$, 1967) führte Untersuchungen zur Populationsdynamik von Daphnia pulex bei chronischer Einwirkung subletaler Dosen einer $\gamma$-Strahlung durch. ENGel (1967) prüfte die Wirkung einmaliger und chronischer Bestrahlungen auf Uberlebenszeit und Wachstum 
von Callinectes sapidus. ENGEL et al. (1966) fanden Beziehungen zwischen Bestrahlung und Atmungsgröße bei Artemia salina. White et al. (1967), ANgelovic et al. (1966) und ANGELOvic \& ENGEL (1968) untersuchten die Zusammenhänge zwischen Salinität, Temperatur und Strahlenwirkung bei Artemia salina und Palaemonetes pugio.

Eine detaillierte Arbeit über den Einfluß einer Bestrahlung auf das Häutungsgeschehen fehlte bisher. An Hand eines größeren Versuchsmaterials wird in der vorliegenden Untersuchung dargelegt, welche Wirkung eine einmalige Röntgenbestrahlung auf die Überlebenszeit und die Häutungsvorgänge bei Gammariden hat. Die Gammariden wurden wegen ihrer Bedeutung als Nahrungstiere, insbesondere für Fische, gewählt, und weil sie leicht in ausreichender Menge beschafft werden können. Auch stößt ihre Hälterung im Laboratorium auf nicht zu große Schwierigkeiten. Die Tiere wurden mit Dosen zwischen 625 und 20000 Röntgen bestrahlt. Die hohen Dosen von mehreren Tausend Röntgen wurden benutzt, um Dosiswirkungskurven über einen größeren Dosisbereich aufstellen und deutlich genug erkennen zu können, wie sich eine Schädigung manifestiert. Aber selbst die niedrigste in den Versuchen verwendete Dosis ist noch hoch gegenüber der Dosis, die bei einer radioaktiven Verseuchung in einem größeren Meeresgebiet erwartet werden kann. Aus technischen Gründen können Experimente mit chronischer Bestrahlung in kontaminiertem Seewasser zur Zeit noch nicht durchgeführt werden. Derartige Versuche, welche die vorliegenden Ergebnisse ergänzen sollen, sind jedoch bereits vorbereitet worden. Sie verfolgen das Ziel, die langfristige Wirkung subletaler Dosen auf die Reproduktionsrate und Populationsdynamik von Gammariden sowie von Copepoden zu prüfen.

\section{MATERIAL UND METHODE}

Gammarus duebeni LiLLJEBORG wurde in Außendeichgräben im Gebiet des Elbeästuars bei Otterndorf gefangen, Gammarus salinus Spooner im inneren Teil der Schwentinemündung (Kieler Förde) und Gammarus zaddacbi Sexton im Nord-OstseeKanal bei Sehestedt. Alle drei Arten leben an den Fangplätzen im mesohalinen Bereich, wobei die größte Abundanz von Gammarus salinus und Gammarus zaddachi in der $\beta$-mesohalinen Zone liegt (Schütz 1964, SchüTz \& KINNE 1955).

Die Bestrahlungen erfolgten nach einer Eingewöhnungszeit von etwa 14 Tagen. Bei Gammarus salinus und Gammarus zaddachi zeigte sich stets nach dem Einbringen der Tiere in das Laboratorium eine vorübergehend hohe Sterblichkeit. Häufig waren nach 14 Tagen nicht mehr genügend Tiere für einen Versuch am Leben; gelegentlich mußte ein Versuch wegen anhaltend hoher Sterblichkeit abgebrochen werden. Auch KINNE (1953a) fand bei Gammarus salinus und Gammarus zaddachi eine höhere Sterblichkeit als bei Gammarus duebeni. Frisch aus See- und Leitungswasser gemischtes Brackwasser wird schlecht vertragen. $\mathrm{Da}$ auch jedes Unsetzen in neues, gealtertes Wasser die Sterblichkeit vorübergehend leicht erhöht, wurde während der Versuche das Wasser nur dann erneuert, wenn es trüb oder sonst stark verunreinigt war. Ein geringfügiger Anstieg des Salzgehaltes während längerer Versuchszeiten wurde in Kauf genommen. Alle Versuche wurden bei einem Salzgehalt von 10\% (Maximalwert: $10,6 \%$; Minimalwert: 9,6\%) durchgeführt. Futterreste und Exkremente wur- 
den regelmäßig entfernt. Gefüttert wurde mit Grünalgen (Enteromorpha), rohen Kartoffelstückchen, gekochtem Muschelfleisch und Herbstlaub von Erlen. Die Fütterungen und Registrierungen der Toten und bei Gammarus duebeni auch der Häutungen erfolgten in Abständen von 2 Tagen. Der Tod wurde festgestellt durch Berühren und leichtes Drücken der Tiere, was bei gesunden Tieren immer eine Fluchtreaktion zur Folge hat. Ein sicheres Kennzeichen des bevorstehenden oder bereits eingetretenen Todes ist auch das Aufhören des Pleopodenschlags.

Die Bestrahlungen wurden mit einem Röntgentherapiegerät bei $200 \mathrm{kV}, 20 \mathrm{~mA}$ und einem Filter von $0,5 \mathrm{~mm} \mathrm{Cu}$ (einschließlich Innenfilter) durchgeführt. Die Dosisleistung betrug $420 \mathrm{R} / \mathrm{min}$ bei einem Fokusabstand von $21 \mathrm{~cm}$. Sie wurde mit einer Fingerhutkammer in Luft gemessen. Die Tiere (jeweils 50 Individuen) befanden sich während der Bestrahlungen - nur knapp mit Wasser bedeckt - in einer flachen Schale von $65 \mathrm{~mm}$ Innendurchmesser. Es folgte ihre Uberführung in temperaturkonstante Räume, wo Gammarus duebeni einzeln in 1-1-Gläsern und Gammarus salinus und Gammarus zaddachi in Gruppen von je 10 Individuen in 5-1-Glasgefäßen bei $15^{\circ} \pm 1^{\circ} \mathrm{C}$ gehalten wurden. In den Kulturräumen herrschte Normaltag mit 12 Stunden Helligkeit und 12 Stunden Dunkelheit. Die Kontrolltiere wurden bei den Bestrahlungen den gleichen Transport- und Temperaturbedingungen wie die Versuchstiere unterworfen. Die Bestrahlungen fanden bei Zimmertemperatur statt.

Ein bis zwei Tage vor einer Bestrahlung wurden die Tiere zur Bestimmung des Alters, des Geschlechts und der Art in einer 0,1\%igen Lösung von Metacain-Methansulfonat (MS 222) in Brackwasser betäubt. Wenn nicht anders vermerkt, sind in den Versuchen stets adulte Tiere verwendet worden. Um festzustellen, wie gut die Reproduzierbarkeit der Ergebnisse ist, wurden in der Regel alle Versuche zweimal mit je 50 Versuchs- und Kontrolltieren durchgeführt. Bei guter Übereinstimmung der Resultate wurden gleiche Versuche zu einem vereinigt. Bei schlechter Übereinstimmung wurden weitere Versuche durchgeführt, und es wurde festgestellt, ob die auftretenden Abweichungen signifikant sind.

\section{UNTERSUCHUNGSERGEBNISSE}

\section{Bestrahlungen mit 625 und 1250 Röntgen}

Dosen von 625 und $1250 \mathrm{R}$ führen nicht, wie es bei höheren Dosen der Fall ist, zum vorzeitigen Tod aller Versuchstiere, sondern bewirken nur eine zeitweise erhöhte Mortalität. Die Abbildungen 1 und 2 zeigen Uberlebenskurven von $\hat{\delta} \hat{\delta}$ und $\phi ㅇ$ von Gammarus duebeni nach Bestrahlungen mit 625 und $1250 \mathrm{R}$. Um die natürliche Sterblichkeit zu eliminieren, ist die Zahl der Uberlebenden in den bestrahlten Gruppen in Prozent der jeweils lebenden Kontrolltiere ausgedrïckt worden. Die Versuche, die nach 148 Tagen abgebrochen wurden, begannen mit je 100 Versuchs- und Kontrolltieren. Während der ersten drei Wochen übersteigt in der Abbildung 1 die Überlebenskurve der $\hat{\partial} \delta$ nach einer Bestrahlung mit $1250 \mathrm{R}$ die 100-0\%-Marke. Es konnte häufiger beobachtet werden, daß nach der Uberführung in die Versuchsgefäße nur bei den Kontrolltieren eine vorübergehend erhöhte Sterblichkeit auftrat, was zu einem Uber- 


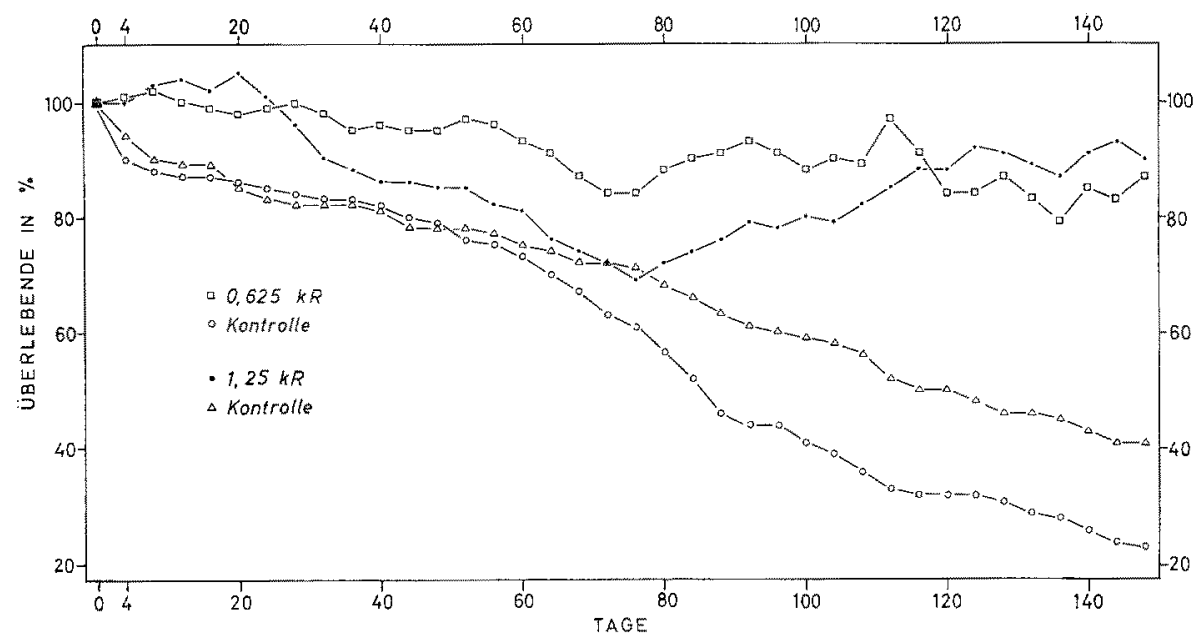

Abb. 1: Oberlebenskurven der $\delta \hat{\delta}$ von Gammarus duebeni nach einmaligen Röntgenbestrahlungen mit 0,625 und $1,25 \mathrm{kR}$. Angaben für bestrahlte Tiere in Prozent der Zahl der jeweils lebenden Kontrolltiere. Anzahl der Tiere pro Versuch: 100

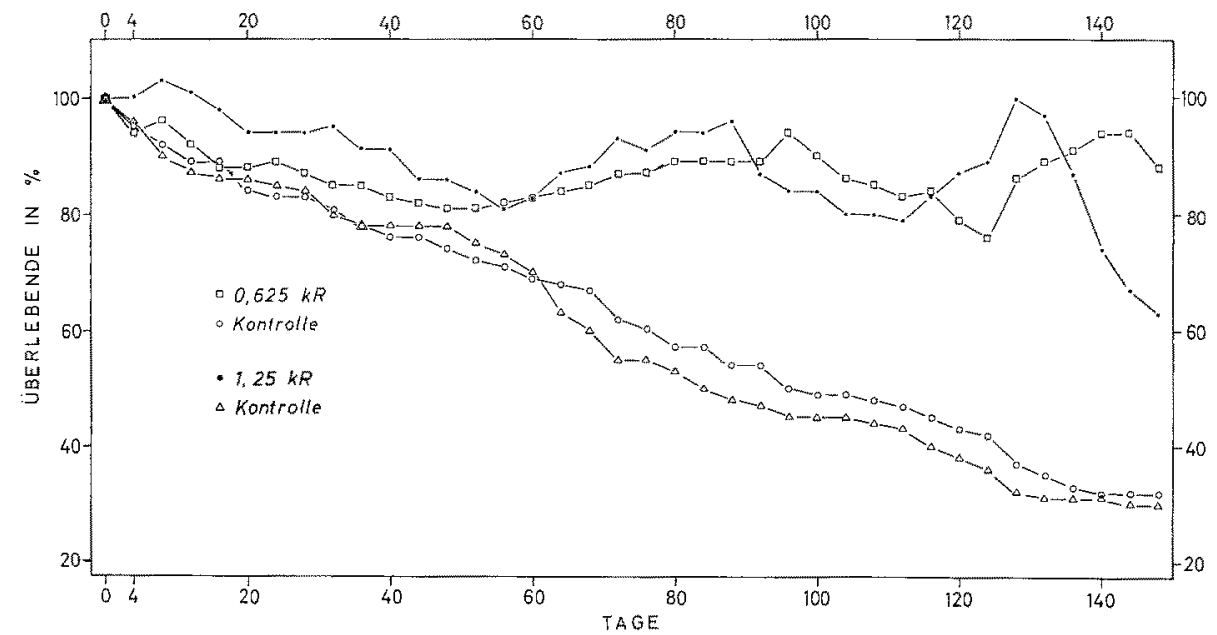

Abb. 2: Uberlebenskurven der 9 von Gammarus duebeni nach einmaligen Röntgenbestrahlungen mit 0,625 und 1,25 kR. Angaben für bestrahlte Tiere in Prozent der Zahl der jeweils lebenden Kontrolltiere. Anzahl der Tiere pro Versuch: 100

steigen der 100-\%-Marke bei den Versuchstieren führen muß, da in dieser Gruppe mehr Tiere am Leben sind als in der Kontrollgruppe. Die Uberlebenskurven erreichen bei den $\delta \partial z$ wischen dem 68. und 80 . Tag und bei den 99 zwischen dem 44. und 60. Tag einen Tiefpunkt. Die Sterblichkeit ist anschließend vorübergehend geringer als in den Kontrollgruppen, bis eine neue Phase erhöhter Sterblichkeit beginnt, die wieder abgelöst wird durch eine Phase geringerer Sterblichkeit. Auch wenn Bestrahlungen mit 


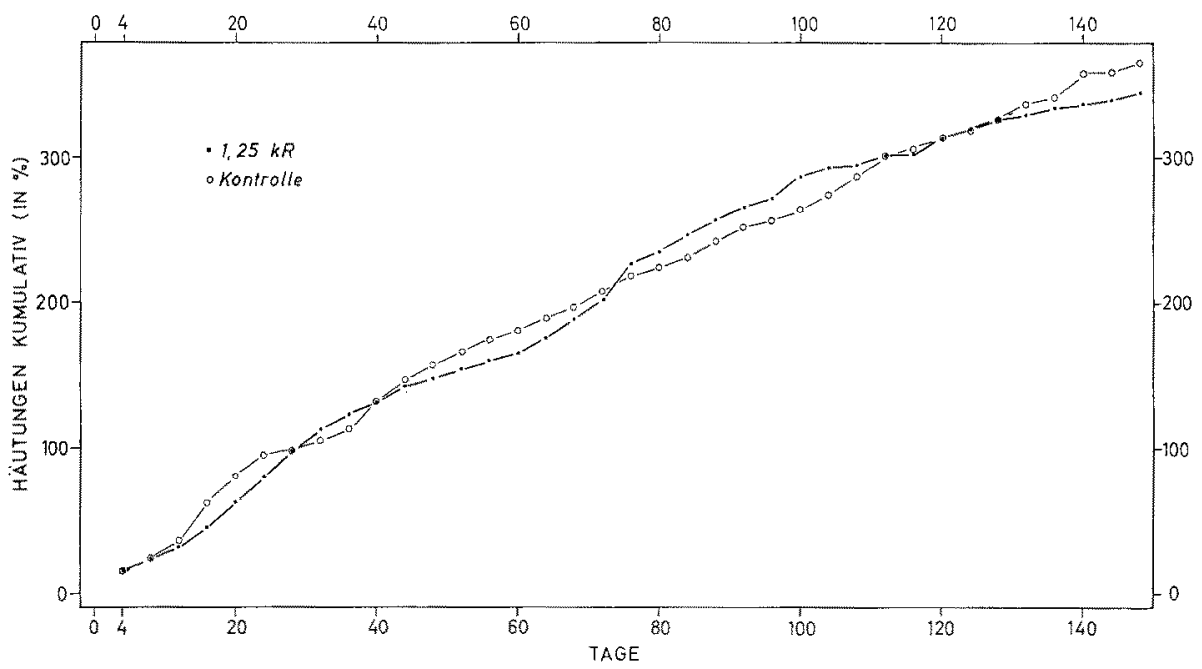

Abb. 3: Anzahl der Häutungen der $\hat{\delta} \hat{\delta}$ von Gammarus duebeni nach einmaliger Röntgenbestrahlung mit 1,25 kR. Häutungen fortlaufend addiert in Prozent der jeweils lebenden Tiere

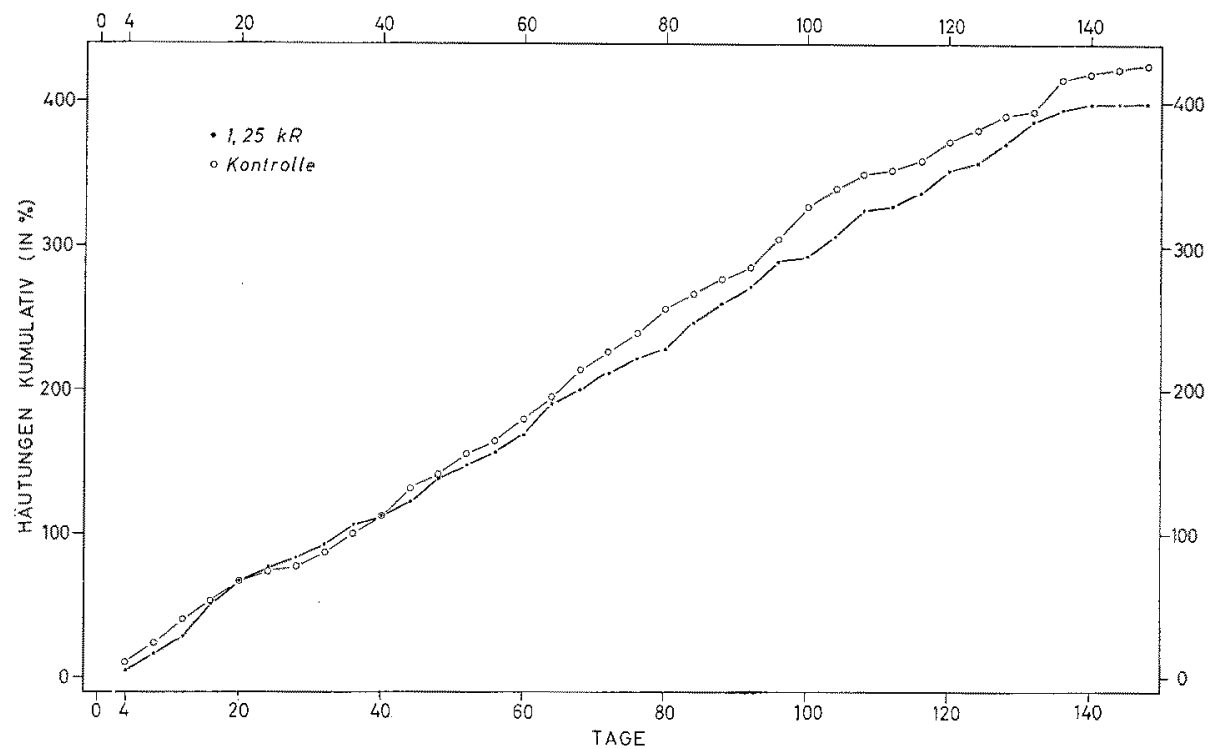

Abb. 4: Anzahl der Häutungen der $q$ 울 von Gammarus duebeni nach einmaliger Röntgenbestrahlung mit 1,25 kR. Häutungen fortlaufend addiert in Prozent der jeweils lebenden Tiere

625 und $1250 \mathrm{R}$ nur zum vorzeitigen Tod einiger Versuchstiere führen, wird doch bereits durch diesen Effekt die Zahl der an der Fortpflanzung teilnehmenden Individuen und damit die Zahl der Nachkommen verringert.

Neben der Zahl der Toten wurden bei Gammarus duebeni auch die Häutungen registriert. Die Abbildungen 3 und 4 zeigen die beobachteten Häutungen bei den Ver- 
suchs- und Kontrolltieren fortlaufend addiert und ausgedrückt in Prozent der jeweils lebenden Tiere. Es zeigen sich keine oder nur geringe Abweichungen der Versuchs- von den Kontrollgruppen nach einer Bestrahlung mit $1250 \mathrm{R}$; die Häutungsfrequenz erfährt keine erkennbare Veränderung. Bunnterm (1965) beobachtete bei den $\hat{\delta} \delta \hat{~ v o n}$ Gammarus duebeni eine stetige Verlängerung des Häutungsintervalls bis zum Erreichen einer Größe von $20 \mathrm{~mm}$. Die flacher werdenden Summenkurven in der Abbildung 3 spiegeln diese gleichmäßige Verlängerung des Häutungsintervalls wider. Nach KINNE (1953b) erreicht das Häutungsintervall bei den $Q$ Q 9 eine definitive Länge mit Eintreten der Geschlechtsreife. Die Kurven der fortlaufend addierten Häutungen zeigen bei den qQ (Abb. 4) eine Abflachung am Ende der Versuchszeit, was auf eine Verlängerung des Häutungsintervalls bei isoliert gehaltenen oder sehr alten $_{5}$ 우 zurückgeführt werden kann (Krnne 1953b), Die höhere Zahl der Häutungen bei den $\$$ o erklärt sich dadurch, daß sie kürzere Häutungsintervalle als die $\delta \hat{\delta}$ aufweisen. Werden $\delta \hat{\delta}$ und $q q$ paarweise bei einer konstanten Temperatur von $15^{\circ} \mathrm{C}$ gehalten, erfolgen bei den $\phi ㅇ$ die Häutungen in Abständen von 21 bis 22 Tagen und bei den $\delta \hat{\delta}(>20 \mathrm{~mm}$ Länge) in Abständen von 34 bis 42 Tagen (Bulnherm 1965). Es sei vermerkt, daß die Bestimmung der Häutungsintervalle aus den abgebildeten Kurven zu falschen Werten führen muß, da des öfteren Häutungen nicht registriert werden konnten, weil entweder die Tiere ihre Exuvien vollständig aufgefressen hatten, oder sie nur Reste übrigließen, die leicht übersehen werden konnten.

\section{Bestrahlungen mit 2000 bis 20000 Röntgen}

Bestrahlungen mit höheren Dosen führen zum vorzeitigen Tod aller Versuchstiere. Die Abbildungen 5 und 6 zeigen die Uberlebenskurven von $\hat{\delta} \delta$ und $9+9$ von Gammarus duebeni nach Bestrahlungen mit 2500, 5000, 10000 und 20000 R. Die Angaben auf den Ordinaten beziehen sich wiederum auf die Zahl der jeweils lebenden Kontrolltiere. Auf die Wiedergabe der Uberlebenskurven der Kontrollgruppen wird hier wie im folgenden verzichtet. Es wurde nicht bei allen Versuchen der Tod auch der letzten Tiere abgewartet, sondern der Versuch vorher abgebrochen, wenn dadurch an Zeit und Platz gespart werden konnte. Die Beurteilung der Strahlenempfindlichkeit erfolgt daher nicht nach den mittleren Uberlebenszeiten, sondern nach den Medianwerten, das sind die Zeiträume, in denen $50 \%$ der Versuchstiere sterben. Mittlere Uberlebenszeit und Medianwert stimmen überein, wenn die Verteilung der individuellen Uberlebenszeiten symmetrisch ist - eine Voraussetzung, die hier nicht erfüllt ist. Der Zeitraum, in dem 50\% der Versuchstiere sterben, verringert sich mit größer werdender Dosis, wobei die Verringerung zwischen 2500 und $5000 \mathrm{R}$ und 10000 und $20000 \mathrm{R}$ einerseits größer ist als $\mathrm{zwischen} 5000$ und $10000 \mathrm{R}$ andererseits.

$A \delta$ und 9 우 von Gammarus zaddachi wurden mit $2000,4000,6000,8000,10000$ und $16000 \mathrm{R}$ bestrahlt (Abb. 7 und 8). Bei diesen und bei den mit 2000, 4000, 8000, 10000 und $16000 \mathrm{R}$ bestrahlten $\delta 3$ und $q 9$ von Gammarus salinus (Abb. 9 und 10) ist deutlicher zu erkennen, daß sich der Zeitraum, in dem 50\% der Tiere sterben, bei mittleren Dosen nur wenig oder gar nicht mit der Dosis ändert. Ein derartiger Konstanzbereich wurde erstmalig von Rajewsky \& Dorneich (vgl. Rajewsky \& Schraub 


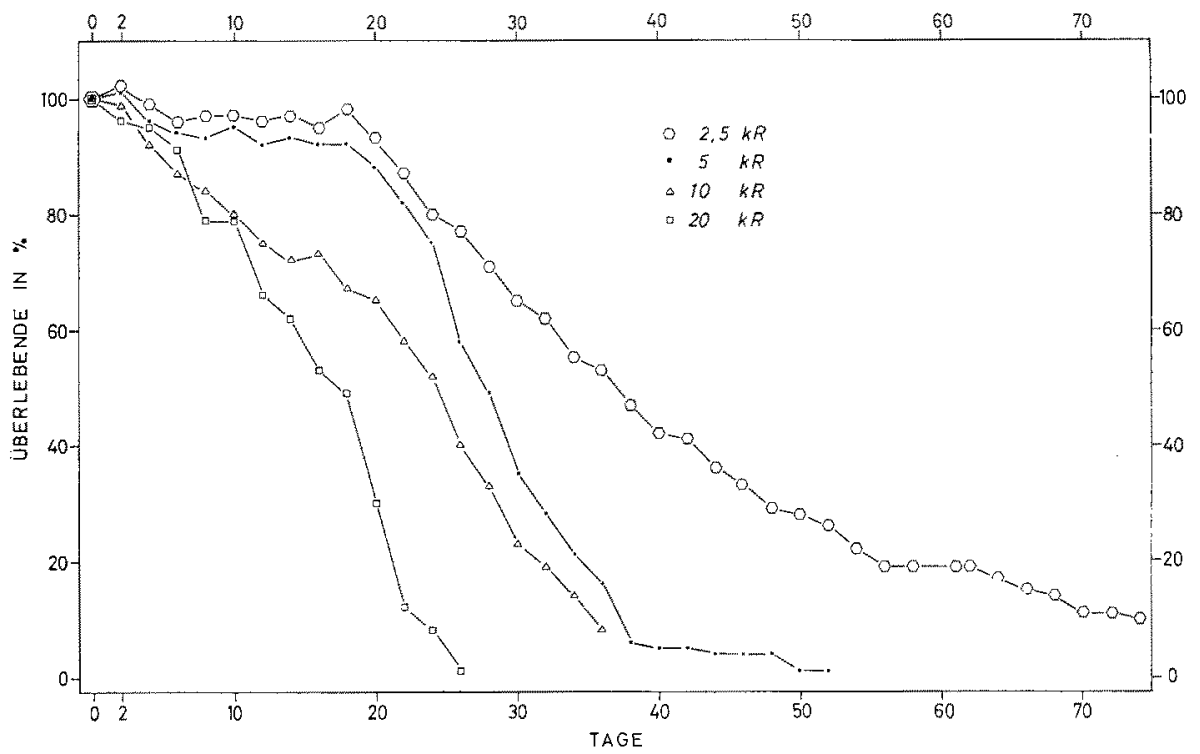

Abb. 5: Uberlebenskurven der $\delta$ f von Gammarus duebeni nach einmaligen Röntgenbestrahlungen. Angaben in Prozent der Zahl der jeweils lebenden Kontrolltiere. Anzahl der Tiere pro Versuch: 100

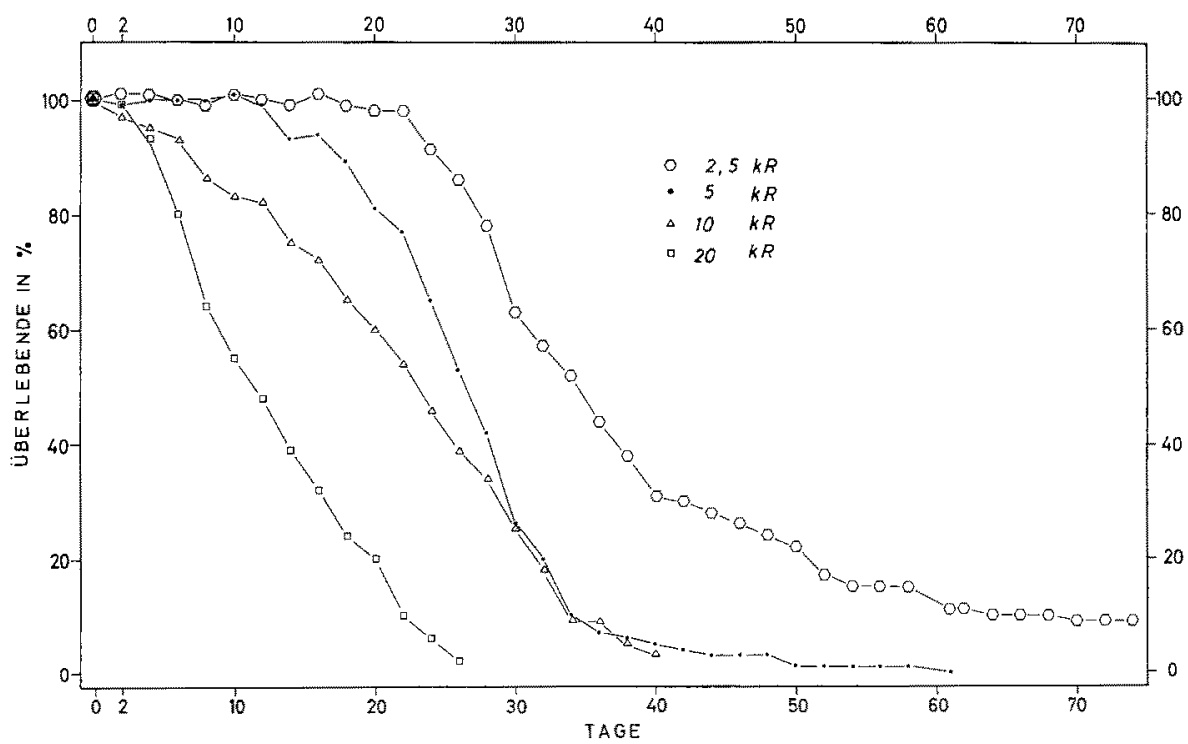

Abb. 6: Uberlebenskurven der $9 \uparrow$ von Gammarus duebeni nach einmaligen Röntgenbestrahlungen. Angaben in Prozent der Zahl der jeweils lebenden Kontrolltiere. Anzahl der Tiere pro Versuch: 100

1948) bei der Albinomaus gefunden. Bei Bestrahlungsdosen zwischen 1200 und $12000 \mathrm{R}$ ändert sich bei dieșem Tier die mittlere Uberlebenszeit nicht mit der Dosis. 


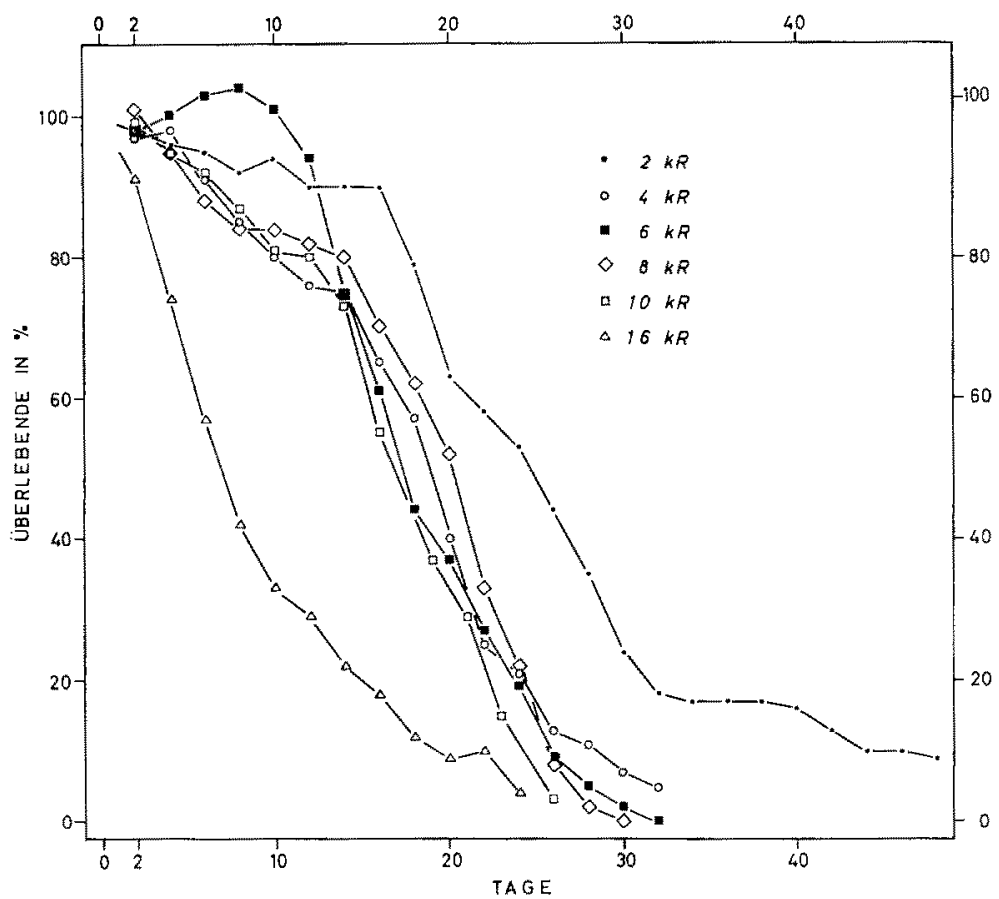

Abb. 7: Oberlebenskurven der ô fo von Gammarus zaddachi nach einmaligen Röntgenbestrahlungen. Angaben in Prozent der Zahl der jeweils lebenden Kontrolltiere. Anzahl der Tiere pro Versuch: 100

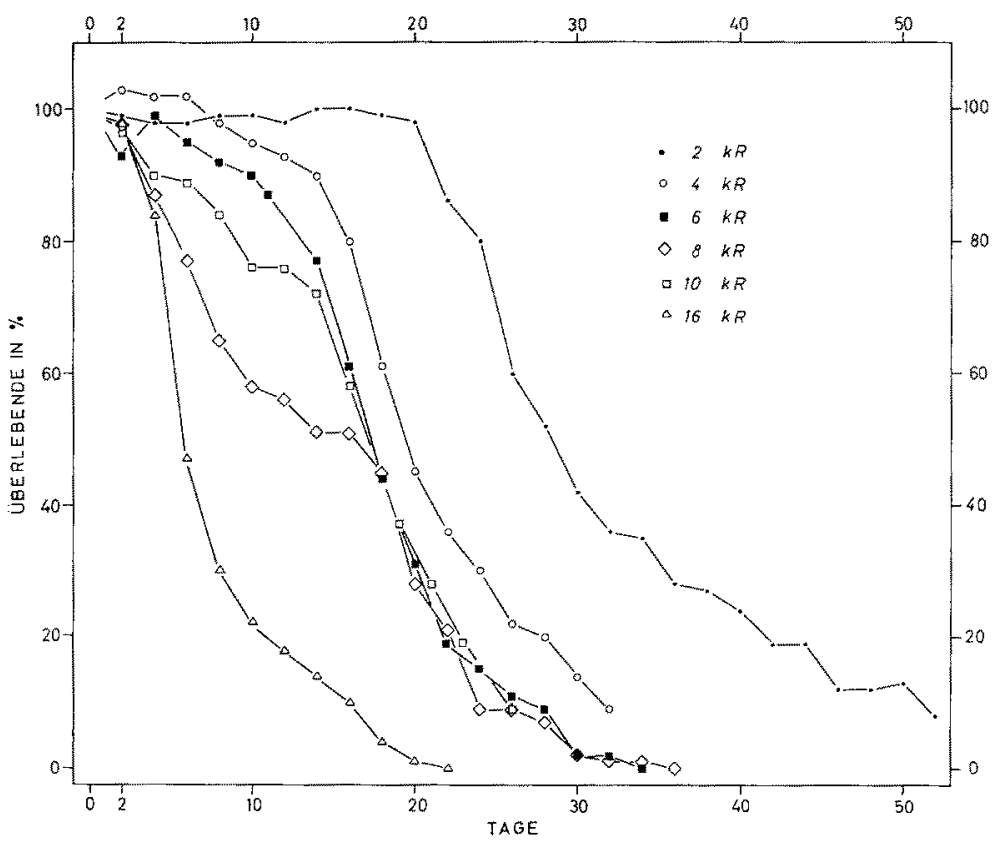

Abb. 8: Uberlebenskurven der $\$ 9$ von Gammarus zaddachi nach einmaligen Röntgenbestrahlungen. Angaben in Prozent der Zahl der jeweils lebenden Kontrolltiere. Anzahl der Tiere pro Versuch: 100 


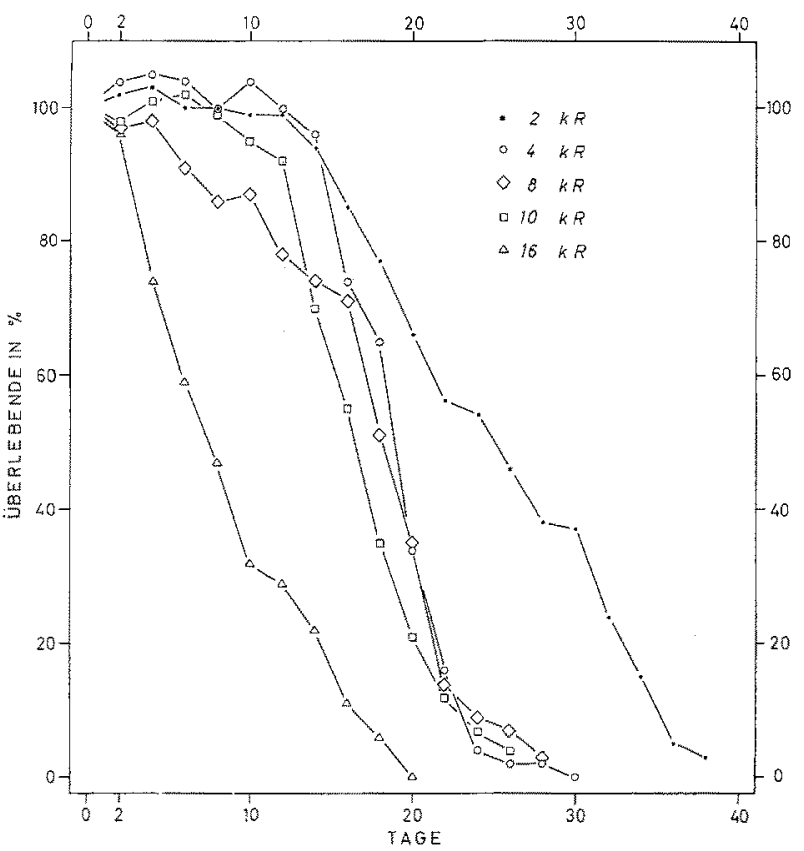

Abb. 9: Uberlebenskurven der $\hat{\delta} \delta$ von Gammarus salinus nach einmaligen Röntgenbestrahlungen. Angaben in Prozent der Zahl der jeweils lebenden Kontrolltiere. Anzahl der Tiere pro Versuch: 100

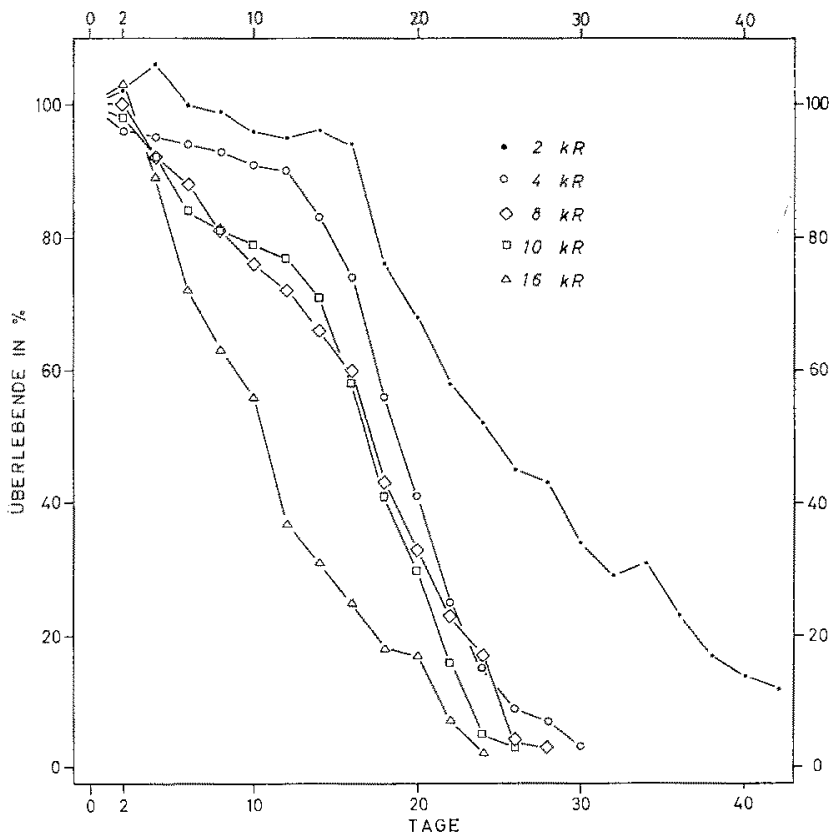

Abb. 10: Uberlebenskurven der $O Q$ von Gammarus salinus nach einmaligen Röntgenbestrahlungen. Angaben in Prozent der Zahl der jeweils lebenden Kontrolltiere. Anzahl der Tiere pro Versuch: 100 


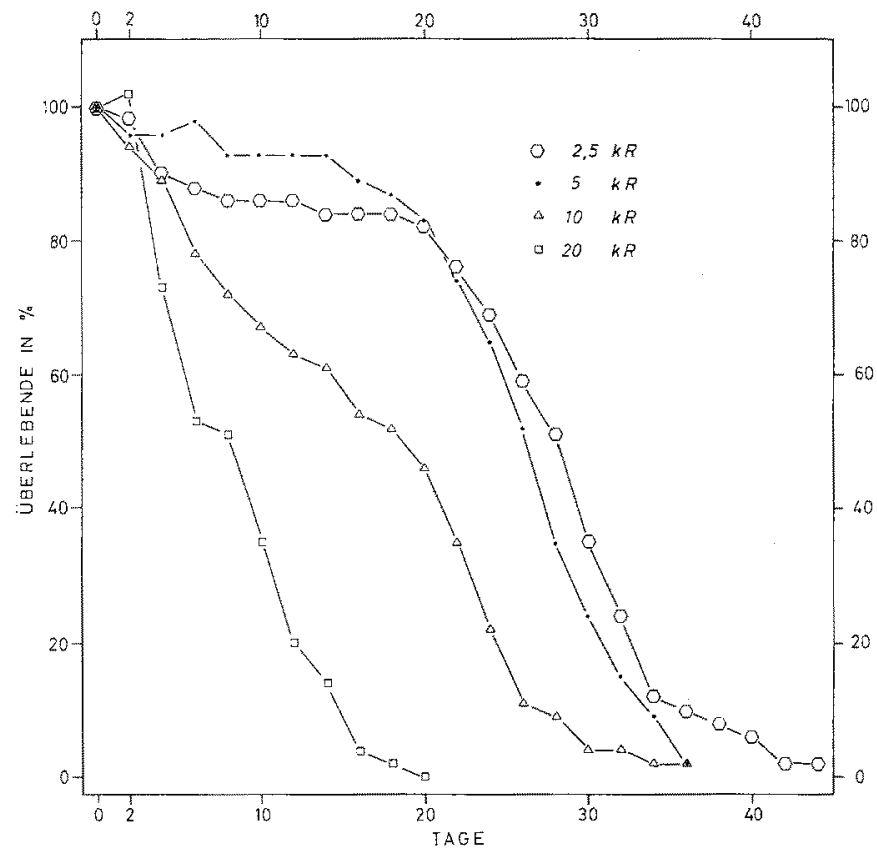

Abb. 11: Überlebenskurven der Jungtiere von Gammarus duebeni nach einmaligen Röntgenbestrahlungen. Angaben in Prozent der Zahl der jeweils lebenden Kontrolltiere. Anzahl der Tiere pro Versuch: 50 (aus Laboratoriumszuchten)

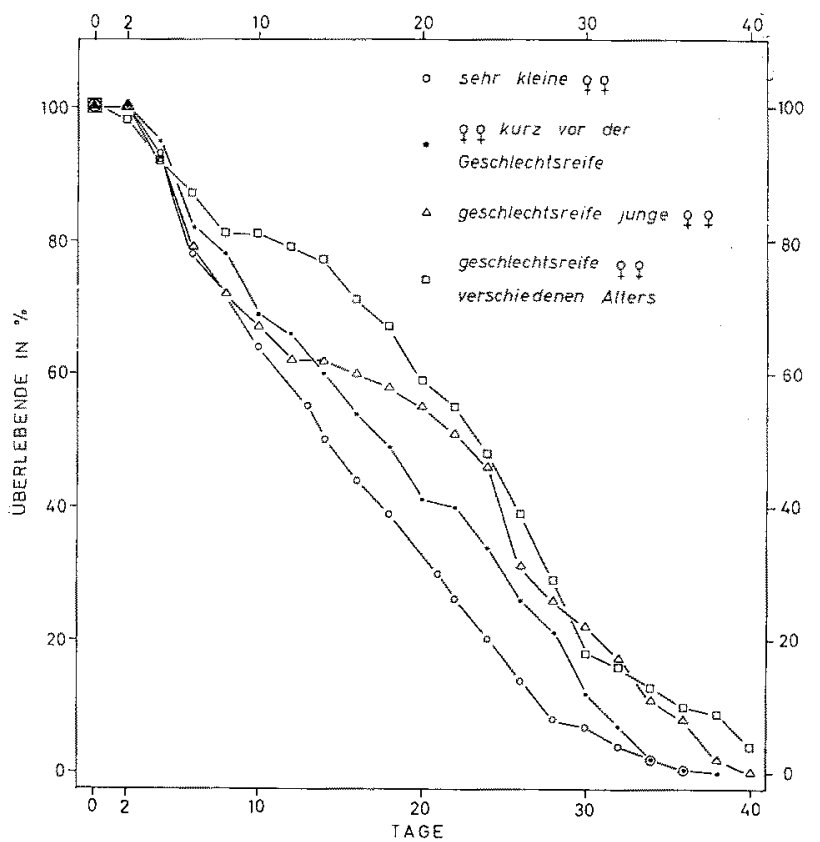

Abb. 12: Uberlebenskurven von 우 verschiedenen Alters nach einmaliger Röntgenbestrahlung mit $10 \mathrm{kR}$. Angaben in Prozent der $\mathrm{Zahl}$ der jeweils lebenden Kontrolltiere. Anzahl der Tiere pro Versuch: 100 (우 von Gammarus duebeni aus Laboratoriumszuditen) 
Die Abbildung 11 zeigt Uberlebenskurven von Jungtieren von Gammarus duebeni. Es handelt sich um Tiere von 4 bis $7 \mathrm{~mm}$ Länge aus Laboratoriumszuchten. Eine Unterscheidung von $\hat{\partial} \delta$ und $\phi Q q$ ist bei dieser Größe noch nicht möglich. Die Unterschiede zwischen den Uberlebenskurven der Jungtiere und der adulten Tiere (siehe Abb. 5 und 6) nach Bestrahlungen mit 2500, 10000 und $20000 \mathrm{R}$ sind nach dem KolmogoROFF-SMIRNofF-Test für zwei unabhängige Stichproben (vgl. SACHs 1968) mit 0,1 bis $1 \%$ Irrtumswahrscheinlichkeit gesichert. Nach Bestrahlungen mit $5000 \mathrm{R}$ sind die Uberlebenskurven der jungen und adulten Tiere fast gleich. In Abbildung 12 sind Überlebenskurven nach Bestrahlungen mit $10000 \mathrm{R}$ für $\nmid 9$ verschiedenen Alters zusammengestellt worden. Alle Tiere stammen aus Laboratoriumszuchten. Bei den kurz vor der Geschlechtsreife stehenden und geschlechtsreifen jungen $O 9+$ handelt es sich um Tiere aus mehreren gleichzeitig produzierten Würfen. Die geschlechtsreifen jungen $q$ q sind 7 Wochen älter als die kurz vor der Geschlechtsreife stehenden. Die Zeiten, in denen $50 \%$ der geschlechtsreifen jungen $\$$ 우 und $50 \%$ der nicht ausgesuchten adulten 우 sterben, sind fast gleich. Der Unterschied $z$ wischen den Uberlebenskurven der geschlechtsreifen jungen $ㅇ ㅜ$ und sehr kleinen $q$ 우 ist nach dem Kolmogoroff-SMIRNOFFTest mit 1\% Irrtumswahrscheinlichkeir gesichert. Da die Strahlenresistenz der + sich nur bis zum Erreichen der Geschlechtsreife ändert, braucht auf die Alterszusammensetzung einer Versuchsgruppe von adulten Tieren nicht besonders geachtet zu werden. $\mathrm{Da}$ auch die Überlebenskurven für nicht ausgesuchte adulte 우우 aus dem Laboratorium und dem Freiland (siehe Abb. 6) nahezu identisch sind, hätten die in dieser Arbeit überwiegend mit Freilandtieren durchgeführten Versuche auch mit Tieren aus Laboratoriumszuchten durchgeführt werden können.

Zur Kennzeichnung der Strahlenempfindlichkeit eines Organismus wird allgemein die $\mathrm{LD}_{50 / 30}$ herangezogen, das ist die Letaldosis, bei der $50 \%$ der Tiere innerhalb von 30 Tagen nach einer Bestrahlung sterben. Voraussetzung für die Benutzung der $L_{50} / 30$ ist eine entsprechend große Lebensspanne der untersuchten Organismen. Die Zeitspanne von 30 Tagen ist gewählt worden, weil sich gezeigt hatte, daß Säuger, die nach einer Ganzkörperbestrahlung diese Zeitspanne überleben, eine gute Chance haben, dem akuten Strahlentod zu entgehen. Doch nicht immer liegt die Grenze für die akute Sterblichkeit bei 30 Tagen. Für die Schlammschnecke Nassarius obsoletus und den See-

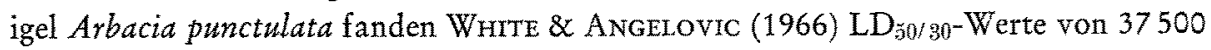
beziehungsweise $38900 \mathrm{R}$, was auf eine etwa gleich große Strahlenempfindlichkeit dieser Arten hinweist. Doch ist die Sterblichkeit bei Arbacia punctulata zwischen dem 30. und 40. Tag höher als bei Nassarius obsoletus, und nad den $L_{50 / 40}$-Werten ist die Schnecke doppelt so strahlenresistent wie der Seeigel. Zum Zwecke einer genaueren Analyse empfiehlt sich daher die Aufstellung vollständiger Dosiswirkungskurven.

Abbildung 13 zeigt die Anderung der $\mathrm{LD}_{50}$ mit der Zeit bei $\delta \hat{\partial}$,, 9 und Jungtieren von Gammarus duebeni. Die Kurven sind im doppellogarithmischen Maßstab aufgetragen, wobei die Ordinate den Logarithmus der Zeit in Tagen bis zum Tode von $50 \%$ der Versuchstiere und die Abszisse den Logarithmus der Dosis in $\mathrm{kR}$ trägt. Aus der Darstellung geht hervor, daß die $\delta$ of strahlenresistenter als die $ㅇ q$, und diese wiederum resistenter als die Jungtiere sind. Es ist ferner zu erkennen, daß die $Q O+$ auf eine höhere Dosis empfindlicher als die of of reagieren. Bei den 우 führt eine um $32 \%$ 
niedrigere Dosis als bei den $\partial \partial$ innerhalb von 15 Tagen und eine nur um $10 \%$ niedrigere Dosis innerhalb von 30 Tagen zum Tode von $50 \%$ der Versuchstiere.

Die Kurven zeigen einen unstetigen Verlauf, der drei Phasen erkennen läßt. Bei hohen Dosen stirbt die Hältte der Tiere bereits innerhalb weniger Tage, bei mittleren Dosen ist die Zeit, in der 50\% der Tiere sterben, dosisunabhängig und liegt zwischen 25 und 26 Tagen. Wir haben es hier mit dem bereits erwähnten Konstanzbereich zu tun. Bei den Jungtieren ist der Bereich zu niedrigeren Dosen hin verschoben. Im Anschluß an den dosisunabhängigen Bereich verlängert sich der Zeitraum, in dem 50\% der Tiere sterben, mit kleiner werdender Dosis, bis bei $1250 \mathrm{R}$ ein Bereich erreicht wird, in dem nur noch wenige der erwachsenen Tiere vorzeitig sterben.

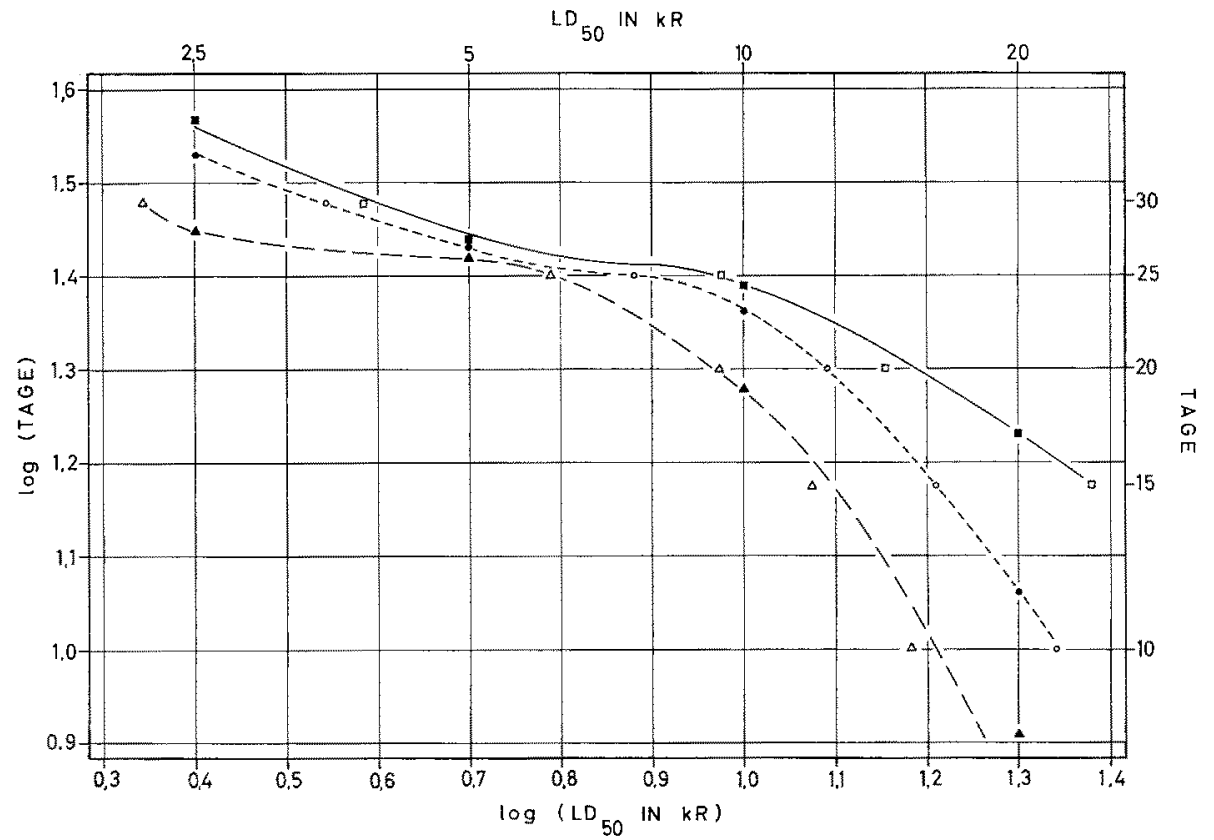

Abb. 13: Dosis-Zeit-Kombinationen, bei denen 50\% der Tiere sterben. Jeder aufgetragene Wert gibt an, bei welcher Dosis in welcher Zeit 50\% der Tiere sterben. $\partial \hat{\delta}(\square), q q(O)$ und Jungtiere $(\triangle)$ von Gammarus duebeni. (Offene und geschlossene Symbole siehe Text)

Der in Abbildung 13 dargestellte Zusammenhang zwischen der für 50\% der Tiere tödlichen Dosis und der Zeit wurde nicht auf rechnerischem, sondern auf graphischem Wege ermittelt. Die von ENGel (1967), White \& ANGelovic (1966), ReEs (1962) und Bonham \& Palumbo (1951) benutzten Schätzverfahren von Reed \& MUENCH (1938) und Irwin \& Cheeseman (1939) sind nicht geeignet, weil durch sie die mittlere wirksame Dosis (im Sinne des arithmetischen Mittels) bestimmt wird, und diese nur dann mit der „50\%ig wirksamen Dosis“ übereinstimmt, wenn die individuell wirksamen Dosen oder deren Logarithmen symmetrisch verteilt sind (vgl. Cavalli-SForza 1964), was bei den untersuchten Gammariden nicht der Fall ist. Aus den gleichen Gründen mußte auch auf die Durchführung einer Probit-Analyse verzichtet werden. 
Am Beispiel der 90 von Gammarus zaddachi soll die Bestimmung der $\mathrm{LD}_{50^{-}}$ Werte erläutert werden. Die Abbildung 14 enthält die für den Dosisbereich von 2000 bis $16000 \mathrm{R}$ ermittelten $\mathrm{LD}_{50}$-Werte für $\delta \delta$ und $q q$ von Gammarus zaddachi. Zur Gewinnung dieser Werte bei den 9 \% wurden die Oberlebenskurven der Abbildung 8 in ein Wahrscheinlichkeitsnetz mit logarithmisch geteilter Abszissenachse übertragen.

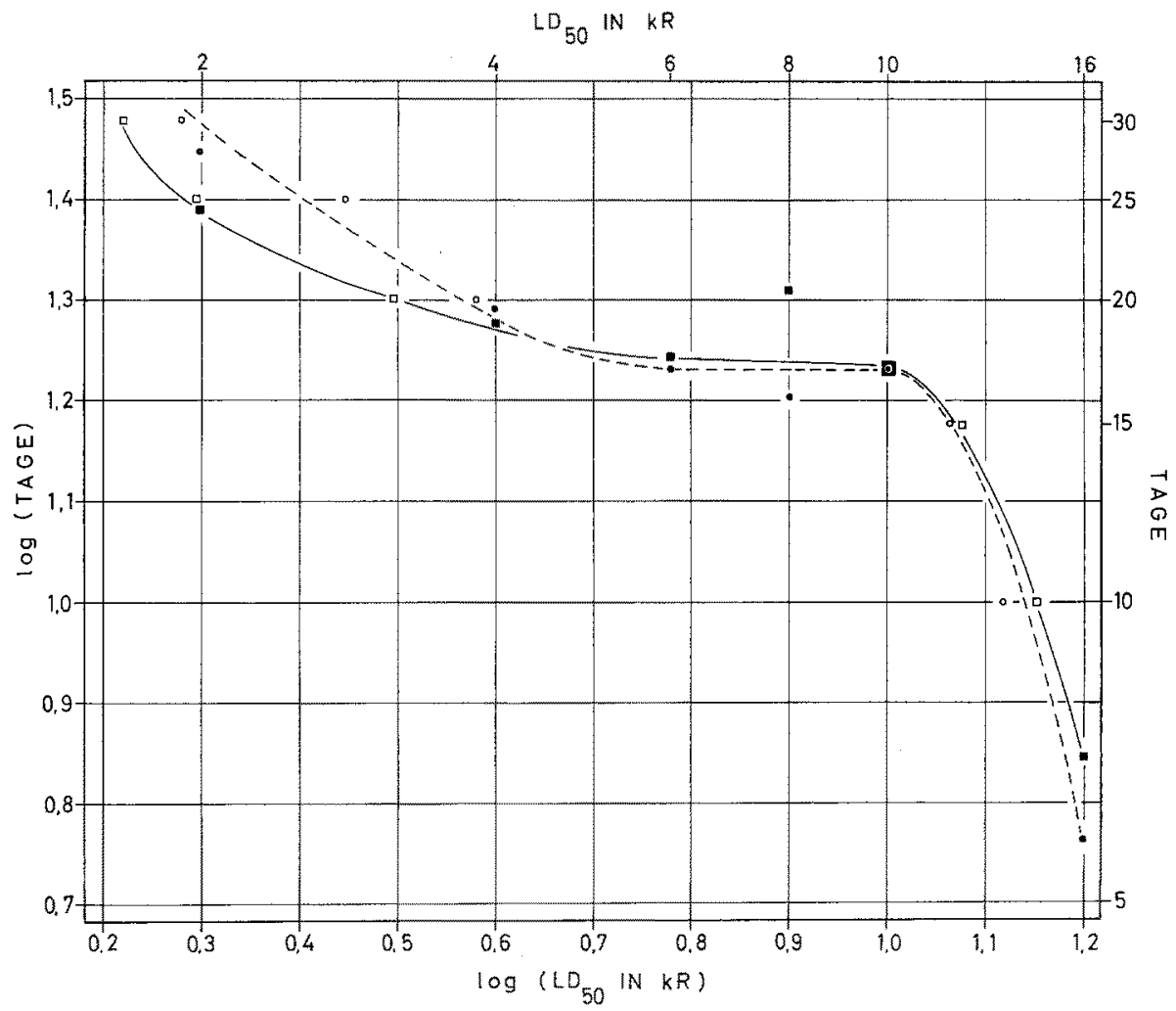

Abb. 14: Dosis-Zeit-Kombinationen, bei denen 50\% der Tiere sterben. Jeder aufgetragene Wert gibt an, bei welcher Dosis in welcher Zeit $50 \%$ der Tiere sterben. $\delta 8$ (D) und $Q 9(0)$ von Gammarws zaddachi. (Offene und geschlossene Symbole siehe Text zu Abb. 13)

Dieses Netz wurde gewählt, weil sich in ihm der zeichnerische Ausgleich am besten đurchführen ließ. Zunächst wurde abgelesen, in welchen Zeiträumen $50 \%$ der Versuchsticre bei den verschiedenen Dosen gestorben waren. Diese Werte erscheinen in der Abbildung 14 als ausgefüllte Symbole. Dann wurde ermittelt, wieviel Prozent der Versuchstiere nach $10,15,20,25$ und 30 Tagen noch am Leben waren. Die aus diesen Werten gebildeten Dosiswirkungskurven zeigt die Abbildung 15. Da sich die Kurven nicht in Geraden transformieren lassen, mußte ein einfacher Weg zur Bestimmung der $\mathrm{LD}_{50}$-Werte beschritten werden. Als Werte wurden die Schnittpunkte der Verbindungsstrecken mit der 50-0/0-Linie genommen. Sie erscheinen als offene Symbole in der Abbildung 14. Abbildung 16 zeigt den Verlauf der Kurven der „50\% ig wirksamen 


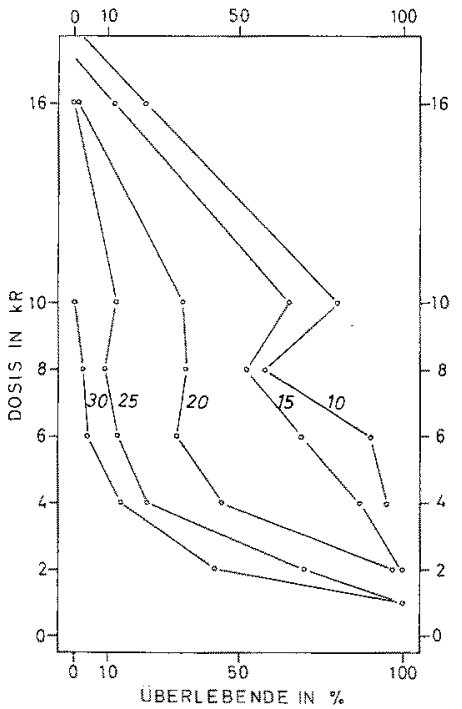

Abb. 15: Dosiswirkungskurven bei 9 우 von Gammarus zaddachi. Aus den Überlebenskurven abgelesene Zahl der Uberlebenden nach 10, 15, 20, 25 und 30 Tagen. Angabe der Úberlebenden in Prozent der jeweils lebenden Kontrolltiere

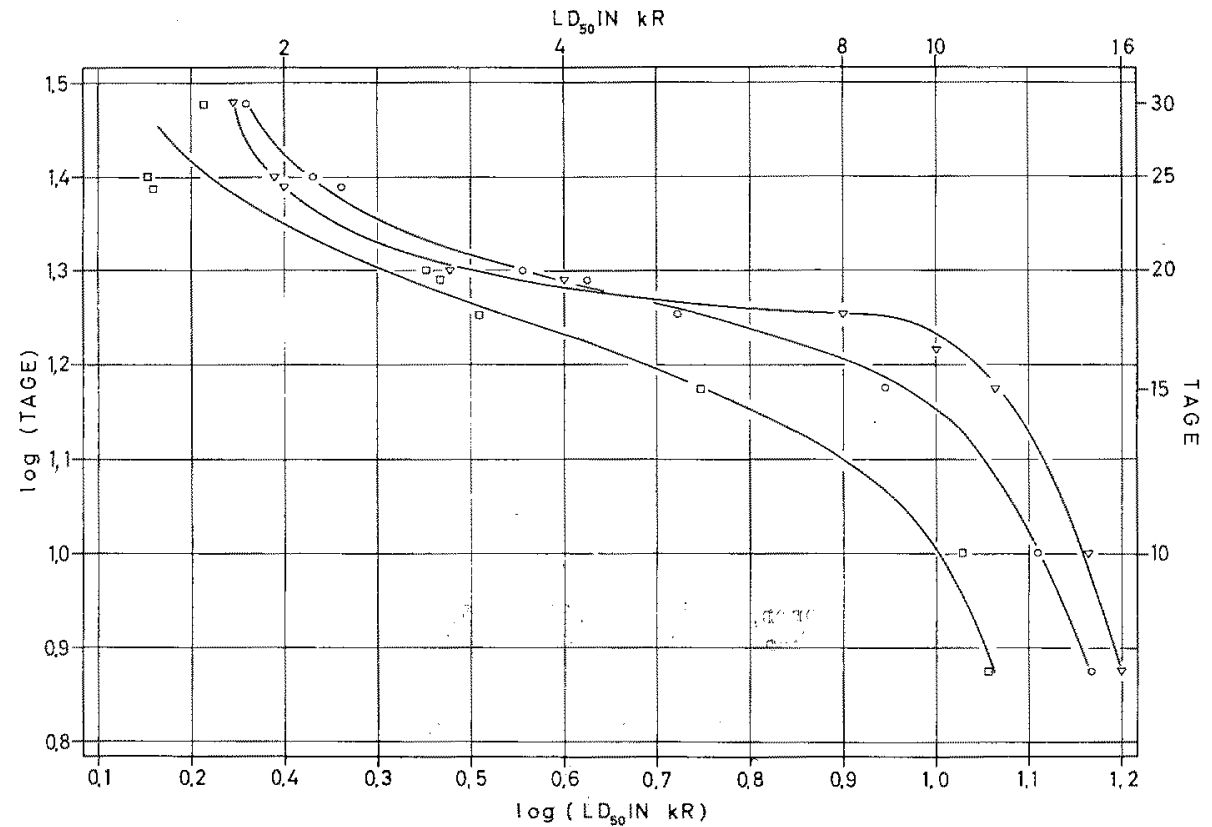

Abb. 16: Dosis-Zeit-Kombinationen, bei denen 50\% der Tiere sterben. $\delta \hat{~} \hat{\theta}$ von Gammarus salinus. Zeichnerisch ermittelte Werte $(\nabla)$, rechnerisch nach IRWIN \& CHIEESEMAN (1939) (O)

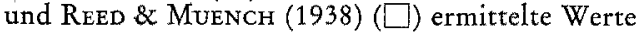


Dosis" bei ô ô von Gammarus salinus, wenn die Schätzverfahren für die mittlere wirksame Dosis von Reed \& Muench (1938) und Irwin \& Cheeseman (1939) benutzt werden. Die Rechnungen ergaben bei den $\hat{\delta} \hat{\delta}$ von Gammarus salinus die beste Ubereinstimmung mit den graphisch ermittelten Werten, dennoch weichen die nach den verschiedenen Verfahren ermittelten Werte stark voneinander ab. Der Konstanzbereich verschwindet bei der Anwendung der Rechenverfahren völlig.

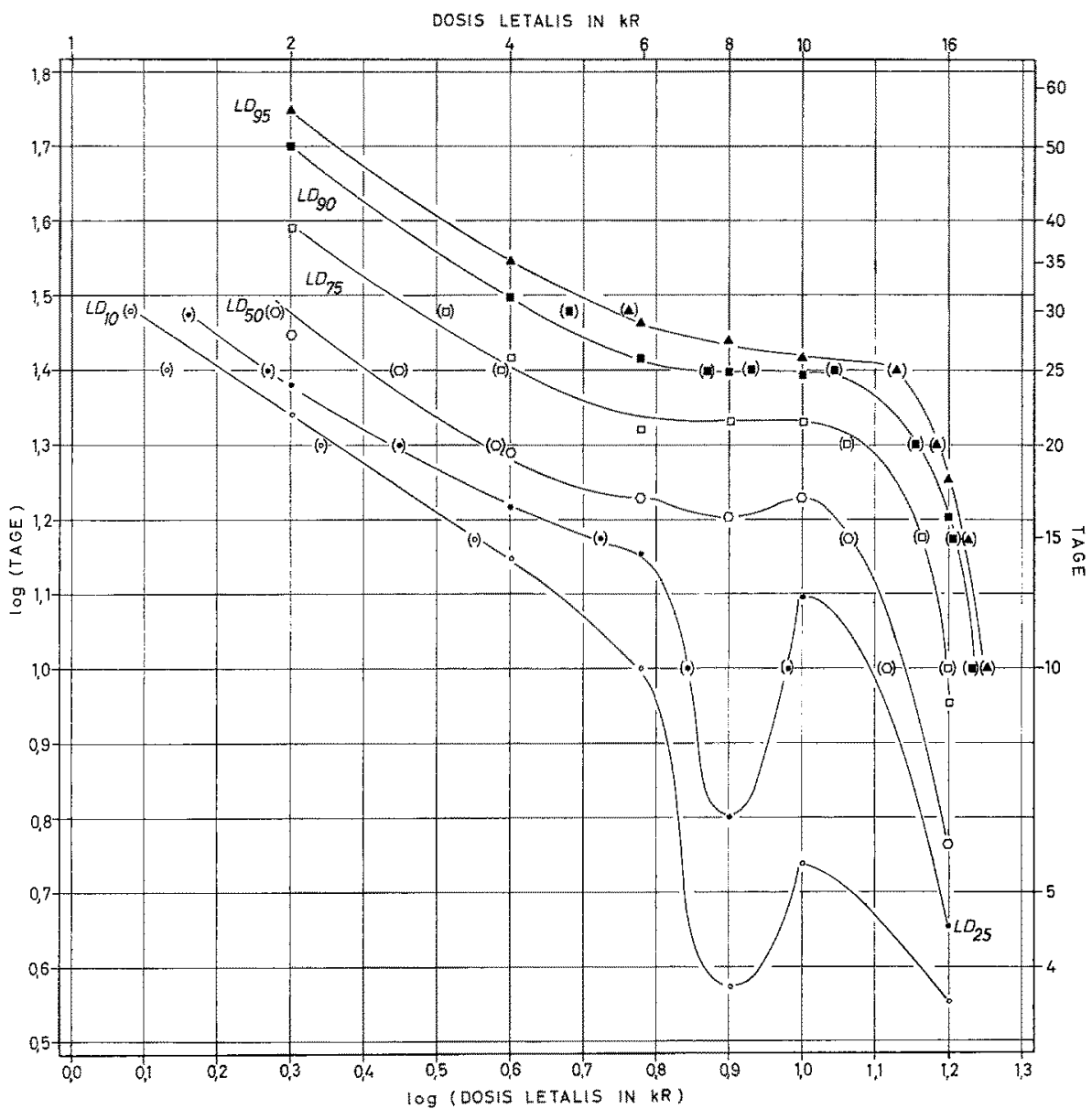

Abb. 17: Dosis-Zeit-Kombinationen, bei denen 10, 25, 50,75, 90 und $95 \%$ der $q 9$ von Gammarus zaddachi sterben. (In Klammern gesetzte Symbole siehe Text)

Die Kurven der Abbildung 15 lassen einen Konstanzbereich zwischen 6000 und $10000 \mathrm{R}$ erkennen, wobei nach 20 Tagen stets etwa 60 bis $65 \%$, nach 25 Tagen etwa 80 bis $90 \%$ der Versuchstiere gestorben sind. Abbildung $8 \mathrm{zeigt}$, daß die Uberlebenskurven für 6000 und $10000 \mathrm{R}$ am 14. Tag zusammenrücken, und erst am 18. Tag die Kurve für $8000 \mathrm{R}$ hinzukommt. Entsprechend finden wir in der Abbildung 15, daß die Kurve der Überlebensprozentsätze nach 15 Tagen bei $8000 \mathrm{R}$ einen Einschnitt be- 
sitzt, während nach 20 Tagen die Überlebensprozentsätze für 6000,8000 und $10000 \mathrm{R}$ gleich sind. Ermittelt man nicht nur die $\mathrm{LD}_{50^{-}}$, sondern auch die $\mathrm{LD}_{10^{-}}, \mathrm{LD}_{25^{-}}, \mathrm{LD}_{75^{-}}$, $\mathrm{LD}_{90^{-}}$und $\mathrm{LD}_{95}$-Werte, so erhält man die in der Abbildung 17 abgebildeten Kurven. Hier stehen die aus den Kurven der Abbildung 15 abgelesenen Werte in Klammern. Ein Konstanzbereich tritt bis hin zur $\mathrm{LD}_{90}$ auf. Die Überlebenskurven der Abbildung 8 zeigen nach Bestrahlungen mit 8000 und $10000 \mathrm{R}$ eine bimodale Verteilung der Todesfälle, wie sie nach Bestrahlungen mit mittleren Dosen auch von Hagen \& Simmons (1947) bei der Ratte und von CoRK (1957) beim Mehlkäfer gefunden wurde. Der

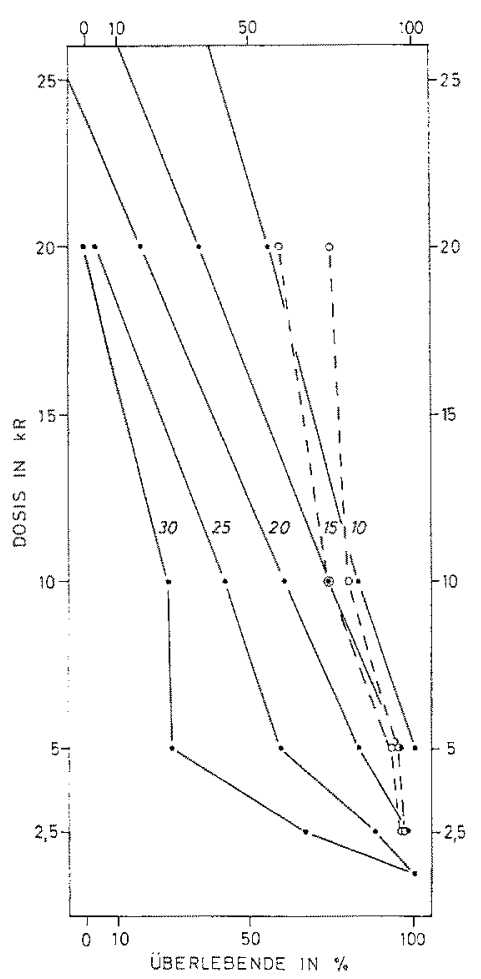

Abb. 18: Dosiswirkungskurven bei Gammarus duebeni. Aus den Oberlebenskurven abgelesene Zahl der Uberlebenden nach 10, 15, 20, 25 und 30 Tagen. Angabe der Uberlebenden in Prozent der jeweils lebenden Kontrolltiere. Ausgezogene Linien: $+\propto+$; gestrichelte Linien: $\hat{\delta} \hat{O}$

bimodale Verlauf der Sterblichkeit wird besonders deutlich sichtbar bei der Dosis von $8000 \mathrm{R}$ in den Dosis-letalis-Kurven für die $\mathrm{LD}_{10}$ und $\mathrm{LD}_{25} .10$ beziehungsweise $25 \%$ der Tiere sind nach einer Bestrahlung mit $8000 \mathrm{R}$ zu einem früheren Zeitpunkt tot als nach Bestrahlungen mit 6000 und 10000 R. Erst bei der $\mathrm{LD}_{50}$ sind die Uberlebenszeiten nach Bestrahlungen mit 6000,8000 und $10000 \mathrm{R}$ etwa gleich.

Die bimodale Verteilung der Todesfälle nach Bestrahlungen mit mittleren Dosen weist auf die Existenz mindestens zweier Mechanismen hin, deren Schädigung zum Tode führen kann. Kein Tier stirbt in der ersten kritischen Phase nach Bestrahlungen mit niedrigen Dosen. Nach Bestrahlungen mit hohen Dosen gelangen nur wenige Tiere 
in die zweite kritische Periode. Die bimodale Sterblichkeit wurde bei allen untersuchten Gammariden gefunden. Die Dosiswirkungskurve der 99 von Gammarus duebeni zeigt nach 10 Tagen bei $10000 \mathrm{R}$ eine schwache Einknickung (bei den $\partial \partial$ ist sie deutlicher), die auf eine relativ höhere Sterblichkeit in diesem Zeitraum und bei dieser

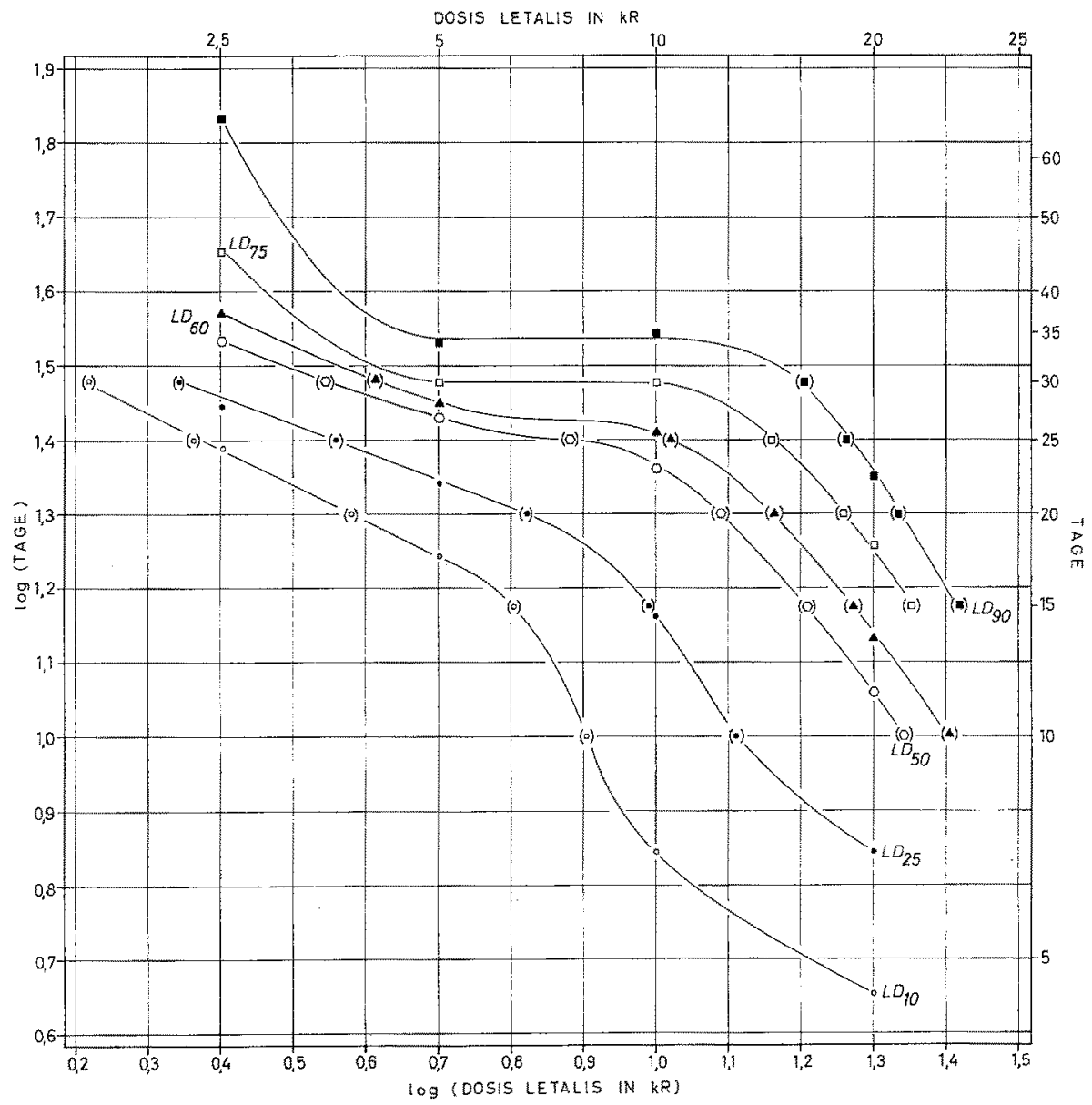

Abb. 19: Dosis-Zeit-Kombinationen, bei denen 10, 25, 50, 60, 75 und $90 \%$ der $9 Q$ von Gammarus duebeni sterben. (In Klammern gesetzte Symbole siehe Text zu Abb. 17)

Dosis hinweist (Abb. 18). In der Abbildung 19 zeigt sich die höhere Sterblichkeit in einem Durchhängen der Dosis-letalis-Kurven für die $\mathrm{LD}_{10}$ und $\mathrm{LD}_{2 \check{\sigma}} \mathrm{zwischen} 6000$ und 20000 R. Gut ausgeprägt ist der Konstanzbereich erst im Verlauf der Dosisletalis-Kurven für die $L D_{75}$ und $L D_{90}$. Wie aus der Abbildung 6 entnommen werden kann, rücken die Uberlebenskurven nach Bestrahlungen mit 5000 und $10000 \mathrm{R}$ auch erst bei einem Uberlebensprozentsatz von $25 \%$ zusammen.

Abbildung 20 zeigt die Abhängigkeit der $L_{50}$ von der Zeit bei $\delta \hat{~} \hat{A}$ und $q q$ von Gammarus salinus. Die Abweichung der $\mathrm{LD}_{50}$-Werte der $\hat{\partial} \hat{o}$ von denen der $q 9$ ist 


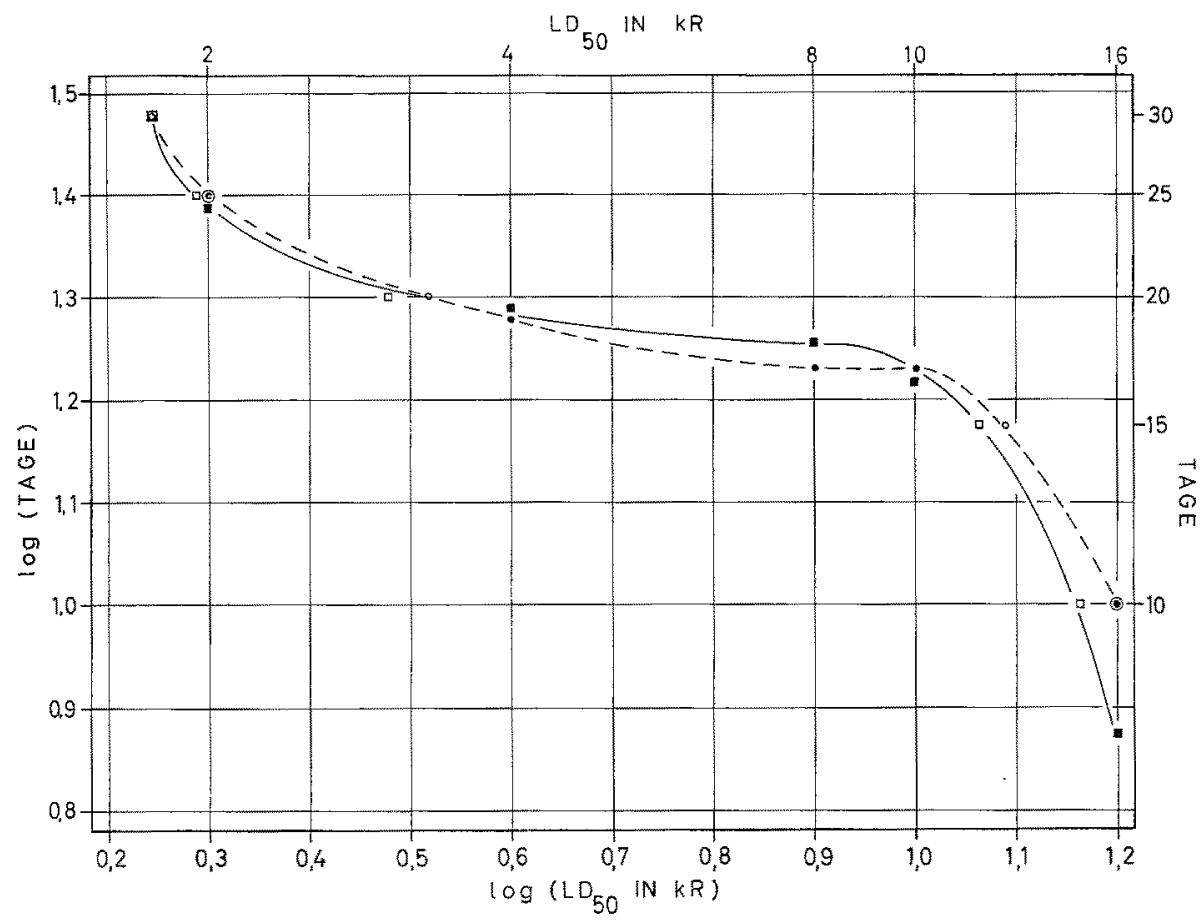

Abb. 20: Dosis-Zeit-Kombinationen, bei denen 50\% der Tiere sterben. Jeder aufgetragene Wert gibt an, bei welcher Dosis in welcher Zeit $50 \%$ der Tiere sterben. $\delta \partial(\square)$ und $q 0$ (O) von Gammarus salinus. (Offene und geschlossene Symbole siehe Text zu Abb. 13)

- wie auch bei Gammarus zaddachi (siehe Abb. 14) - gering. Bei Gammarus zaddachi

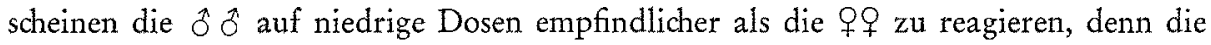
gleiche Sterblichkeit von $50 \%$ innerhalb von 25 Tagen wird bei den $\delta \delta$ durch eine um 29\% niedrigere Dosis als bei den 우오 verursacht. Nicht abgebildete Überlebenskurven von Gammarus salinus und Gammarus zaddachi nach Bestrahlungen mit $1000 \mathrm{R}$ lassen erkennen, daß bei diesen Arten mit $1000 \mathrm{R}$ der Bereich erreicht ist, in dem nicht mehr alle Tiere vorzeitig sterben. In der Tabelle 1 sind die $L D_{50}$-Werte für

Tabelle 1

LD50-Werte (in kR) für Gammarus duebeni, Gammarus salinus und Gammarus zaddachi

\begin{tabular}{|c|c|c|c|c|c|c|c|}
\hline Tage & $\begin{array}{c}\text { G.duebeni } \\
\hat{\delta} \hat{\delta}\end{array}$ & $\begin{array}{c}\text { G. salinus } \\
\delta \delta\end{array}$ & $\begin{array}{c}\text { G. zaddachi } \\
\hat{\delta} \hat{\delta}\end{array}$ & $\begin{array}{l}\mathrm{LD}_{50} \\
\text { G.duebeni } \\
\text { \&9 }\end{array}$ & $\begin{array}{c}\text { G. salinus } \\
\text { o9 }\end{array}$ & $\begin{array}{c}\text { G. zaddachi } \\
\text { 우우 }\end{array}$ & $\begin{array}{l}\text { G.duebeni } \\
\text { Jungtiere }\end{array}$ \\
\hline 30 & 3,9 & 1,7 & 1,7 & 3,5 & 1,7 & 1,9 & 2,2 \\
\hline 25 & 9,5 & 2,0 & 2,0 & 7,6 & 2,0 & 2,8 & 6,2 \\
\hline 20 & 14,3 & 3,0 & 3,1 & 12,3 & 3,3 & 3,8 & 9,4 \\
\hline 15 & 24,0 & 11,6 & 11,9 & 16,2 & 12,4 & 11,6 & 11,9 \\
\hline 10 & - & 14,6 & 14,1 & 22,0 & 16,0 & 13,1 & 15,3 \\
\hline
\end{tabular}


die drei untersuchten Gammariden-Arten zum Vergleich zusammengestellt worden. Aus den Daten geht hervor, daß Gammarus salinus und Gammarus zaddachi etwa gleich strahlenempfindlich, aber strahlenempfindlicher als Gammarus duebeni sind. Bei der Betrachtung dieser Werte muß berücksichtigt werden, daß Versuchs- und Kontrolltiere von Gammarus salinus und Gammarus zaddachi nicht einzeln wie bei Gammarus duebeni, sondern in Gruppen zu je 10 Individuen gehalten wurden, und Kannibalismus auftrat. Häufig waren die Leichen der Flohkrebse angefressen worden, mitunter auch gänzlich verschwunden, doch konnte nicht entschieden werden, ob frisch gehäutete oder bereits tote Tiere an- beziehungsweise aufgefressen wurden. Da die mit höheren Dosen bestrahlten Tiere nach einer gewissen Zeit keine Nahrung mehr aufnehmen, können die ermittelten Ubberlebensprozentsätze, die sich ja auf die Zahl der jeweils lebenden Kontrolltiere beziehen, zu hoch sein. Wenn Kannibalismus auftritt, der die Mortalität nur in den Kontrollgruppen beeinflussen kann, müssen Gammarus salinus und Gammarus zaddachi strahlenempfindlicher sein, als aus der Tabelle hervorgeht. Allerdings konnte - wenn auch selten - bei den Kontrolltieren beider Arten die gleiche Sterblichkeit wie bei den Kontrolltieren der einzeln gehaltenen $\hat{\delta} \hat{\sigma}$ und $q q$ von Gammarus duebeni beobachtet werden.

Die Strahlenempfindlichkeit von Gammarus duebeni scheint im Winter und im Frühjahr von den herrschenden Temperaturen abzuhängen. So zeigten Tiere, die im Januar und März 1967 gefangen und bestrahlt wurden, eine höhere Strahlenresistenz. Die Temperaturen lagen in den Monaten Januar bis April des Jahres 1967 über den langjährigen Monatsmitteln (Tab. 2). Leider liegen keine vergleichbaren Versuche aus

Tabelle 2

Abweichungen der Temperatur $\left({ }^{\circ} \mathrm{C}\right)$ des Oberflächenwassers von den langjährigen Monatsmitteln an der "Alten Liebe" in Cuxhaven. (Nach KüHL 1965-1968)

\begin{tabular}{|lrrrr|}
\hline Monat & 1964 & 1965 & 1966 & 1967 \\
\hline Januar & & $+0,7$ & $-2,6$ & $+0,4$ \\
Februar & & $-0,2$ & $-1,6$ & $+1,7$ \\
März & & $-1,4$ & $+0,5$ & $+2,4$ \\
April & & $-0,4$ & $-2,0$ & $+0,4$ \\
November & $-0,5$ & $-1,9$ & $-0,6$ & \\
Dezember & 0,0 & $-1,7$ & $-1,1$ & \\
\hline
\end{tabular}

anderen Jahren vor. Der Vergleidh der natürlichen Sterblichkeiten in den Jahren 1965 bis 1967 zeigt aber, daß die Tiere in den ersten Monaten des Jahres 1967 eine besonders hohe Widerstandsfähigkeit besessen haben müssen. Die Abbildungen 21 und 22 zeigen Überlebenskurven von $\hat{\partial} \hat{\partial}$ von Gammarus duebeni nach Bestrahlungen mit 10000 und $20000 \mathrm{R}$ im ersten und zweiten Quartal des Jahres 1967. Die Tiere des ersten Quartals zeigen eine deutlich höhere Strahlenresistenz als die des zweiten Quartals. Die Unterschiede sind nach dem Kolmogoroff-Smirnoff-Test (vgl. SAchs 1968) mit 0,1\% Irrtumswahrscheinlichkeit gesichert. In die Abbildung 21 ist zusätzlich die Uberlebenskurve von Tieren aus Laboratoriumszuchten eingetragen worden. Es besteht kein signifikanter Unterschied zwischen ihnen und den im zweiten Quartal bestrahlten 


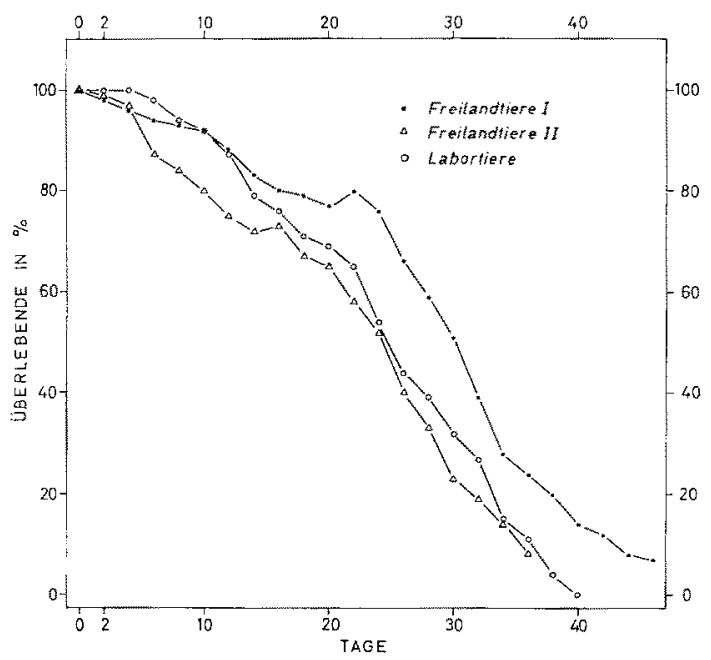

Abb. 21: Oberlebenskurven der $\hat{\partial} \hat{\partial}$ von Gammarus duebeni aus dem Freiland in der ersten Hälfte des Jahres 1967 und aus Laboratoriumszuchten nach einmaliger Röntgenbestrahlung mit 10 kR. Angaben in Prozent der Zahl der jeweils lebenden Kontrolltiere. Freilandtiere I: Bestrahlung im ersten Quartal 1967; Freilandtiere II: Bestrahlung im zweiten Quartal 1967. Anzahl der Tiere pro Versuch: 100

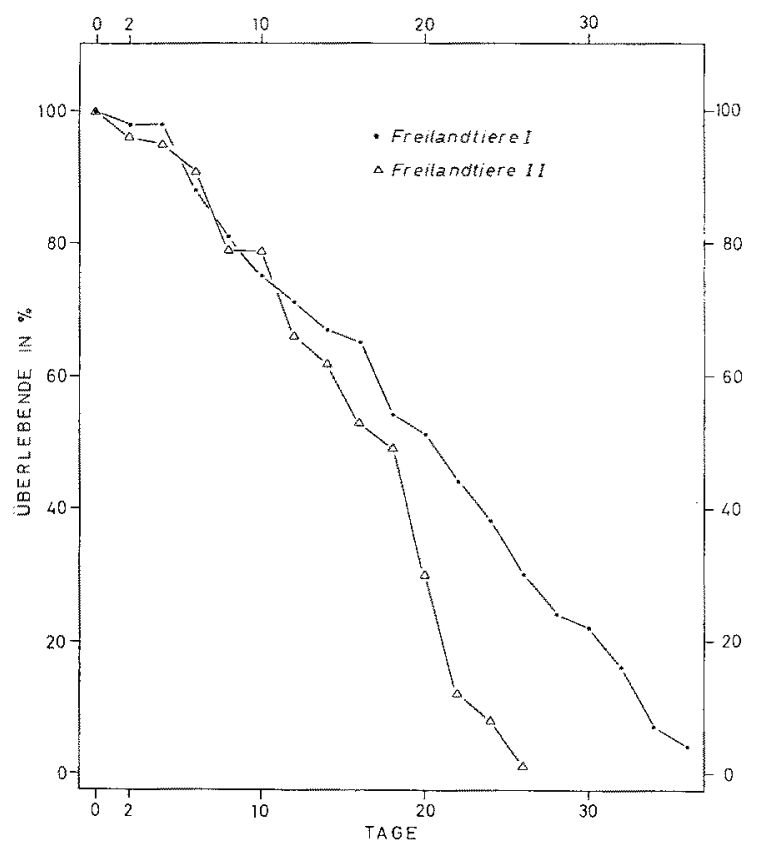

Abb. 22: Uberlebenskurven der $\hat{O}^{*}$ yon Gammarus duebeni aus dem Freiland in der ersten Hälfte des Jahres 1967 nach einmaliger Röntgenbestrahlung mit $20 \mathrm{kR}$. Angaben in Prozent der Zahl der jeweils lebenden Kontrolltiere. Freilandtiere I: Bestrahlung im ersten Quartal 1967; Freilandtiere II: Bestrahlung im zweiten Quartal 1967: Anzahl der Tiere pro Versuch: 100 
Freilandtieren. Auch bei den $9 Q$ ist die Strahlenresistenz im ersten Quartal höher als im zweiten (Abb. 23 und 24). Die Unterschiede zwischen den Überlebenskurven sind wiederum mit 0,1\% Irrtumswahrscheinlichkeit gesichert. Auch hier sind zusätzlich die Überlebenskurven von Tieren aus Laboratoriumszuchten eingetragen worden. Auffallend ist, daß die 90 , die nach Abbildung 13 auf die Dosis von $20000 \mathrm{R}$ empfindlicher reagieren als die $\delta \hat{\delta}$, im ersten Quartal 1967 nach einer Bestrahlung mit

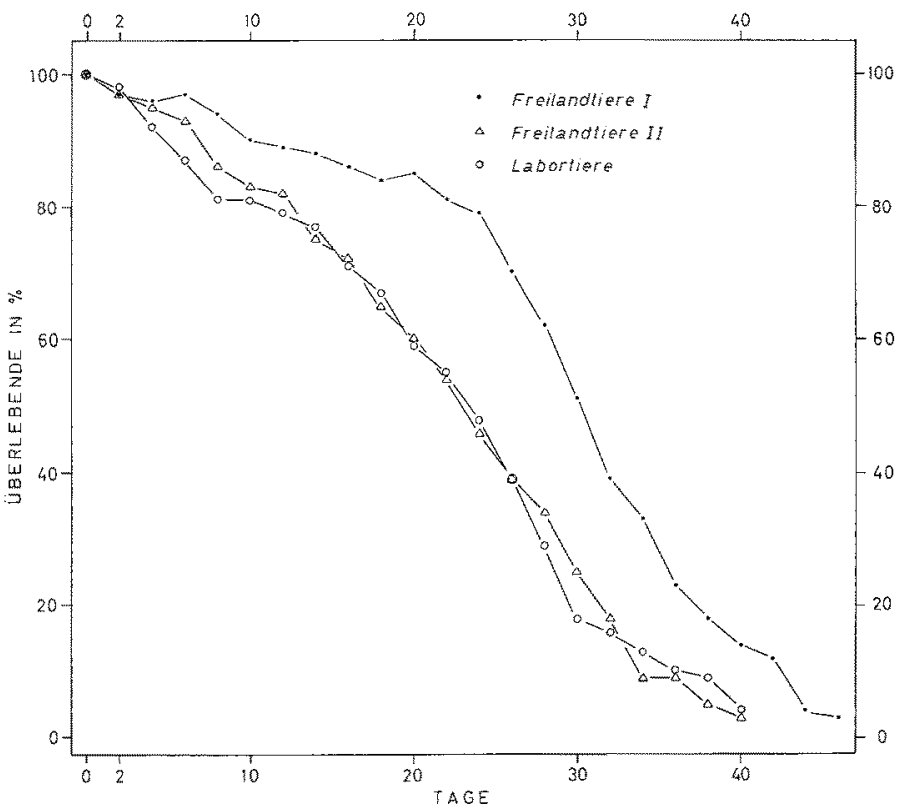

Abb. 23: Überlebenskurven der $q Q$ von Gammarus duebeni aus dem Freiland in der ersten Hälfte des Jahres 1967 und aus Laboratoriumszuchten nach einmaliger Röntgenbestrahlung mit $10 \mathrm{kR}$. Angaben in Prozent der Zahl der jeweils lebenden Kontrolltiere. Freilandtiere I: Bestrahlung im ersten Quartal 1967; Freilandtiere II: Bestrahlung im zweiten Quartal 1967. Anzahl der Tiere pro Versuch: 100

$20000 \mathrm{R}$ später als die $\hat{\delta} \hat{\delta}$ sterben. Der Unterschied zwischen den Überlebenskurven der Tiere des ersten Quartals in den Abbildungen 22 und 24 ist mit $1 \%$ Irrtumswahrscheinlichkeit gesichert. Die natürlichen Sterblichkeiten der $\delta \delta$ und $q$ 우 unterscheiden sich im ersten Quartal nicht, während im zweiten Quartal die strahlenresistenteren $\delta \delta$ eine höhere Kontrollsterblichkeit als die $\phi ㅇ$ zeigen. Eine unterschiedliche Alterszusammensetzung der Versuchsgruppen der $\delta \hat{\delta}$ und $q$ q $q$ im ersten Quartal 1967 kann also für die höhere Strahlenresisten $z$ der $q 9+$ nicht verantwortlich gemacht werden.

\section{Beeinflussung des Häutungsgeschehens}

$\mathrm{Da}$ die Häutungen und die sie begleitenden Vorgänge den Lebenszyklus der Krebse entscheidend beherrschen, ist es naheliegend, diesen periodisch ablaufenden 
Prozessen besondere Aufmerksamkeit zu schenken. Es wurden daher neben den Toten bei Gammarus duebeni auch die Häutungen registriert. Der Versuch, Tiere in genau definierten $\mathrm{Zwischenhäutungsstadien} \mathrm{zu} \mathrm{bestrahlen,} \mathrm{wurde} \mathrm{nicht} \mathrm{unternommen.}$

Wie bereits an anderer Stelle (Abb. 3 und 4) gezeigt wurde, üben Dosen von $1250 \mathrm{R}$ und weniger keine erkennbare Wirkung auf die Häutungsvorgänge aus. Abbildung 25 zeigt die Zahl der Häutungen fortlaufend addiert bei $\hat{\delta} \hat{o}$ von Gammarus

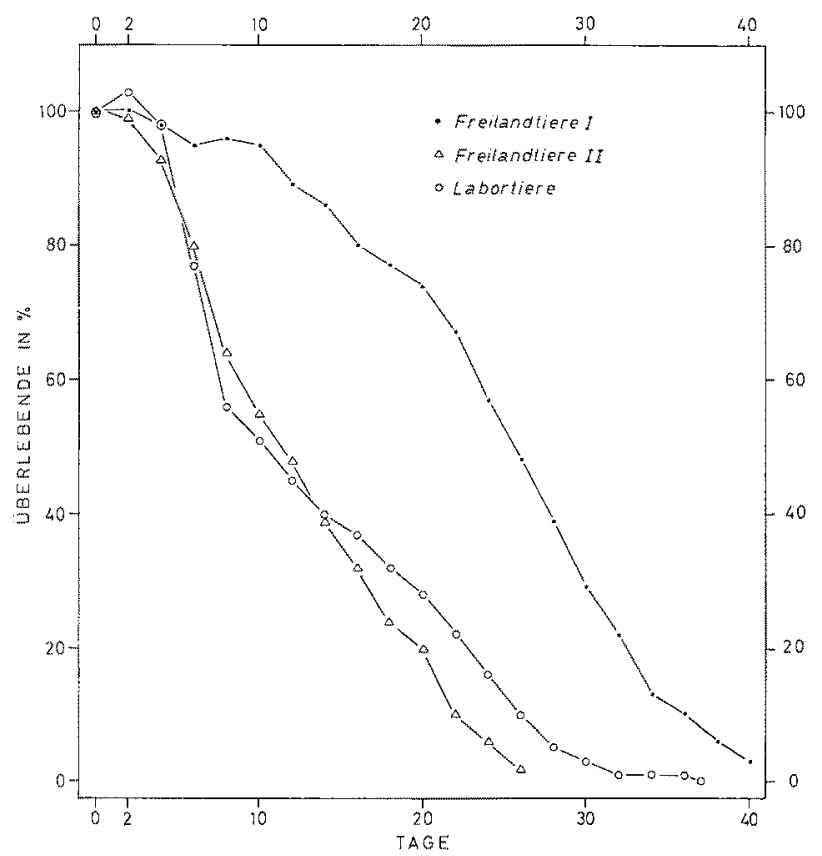

Abb. 24: Überlebenskurven der $Q \bigcirc$ von Gammarus duebeni aus dem Freiland in der ersten Hälfte des Jahres 1967 und aus Laboratoriumszuchten nach einmaliger Röntgenbestrahlung mit $20 \mathrm{kR}$. Angaben in Prozent der Zahl der jeweils lebenden Kontrolltiere. Freilandtiere I: Bestrahlung im ersten Quartal 1967; Freilandtiere II: Bestrahlung im zweiten Quartal 1967. Anzahl der Tiere pro Versuch: 100

duebeni nach Bestrahlungen mit 2500, 5000, 10000 und 20000 R. Die dazugehörigen Überlebenskurven enthält Abbildung 5. Nach einer Bestrahlung mit $20000 \mathrm{R}$ sind nach dem 2. Tag kaum noch Häutungen möglich. Nach $10000 \mathrm{R}$ erfolgen zwischen dem 4. und 6. Tag keine Häutungen. Auch nach $5000 \mathrm{R}$ ist die Zahl der Häutungen in der Versuchsgruppe herabgesetzt. Nach einer Bestrahlung mit $2500 \mathrm{R}$ sind in der Versuchs- und in der Kontrollgruppe bis zum 32. Tag gleich viele Häutungen erfolgt; dann knickt die Häutungssummenkurve der Versuchstiere $a b$ und wird erheblich flacher. Auch bei den mit 5000 und $10000 \mathrm{R}$ bestrahlten Versuchsgruppen werden die Kurven um den 30. Tag herum flacher.

Die Häutungssummenkurven der $q$ enthält die Abbildung 26. Die dazugehörigen Uberlebenskurven finden sich in der Abbildung 6. Auch hier sind Häutungen nach einer Bestrahlung mit $20000 \mathrm{R}$ nur unmittelbar nach Versuchsbeginn möglich. Nach $10000 \mathrm{R}$ erfolgen $z$ wischen dem 2. und 8. Tag keine Häutungen. Nach $5000 \mathrm{R}$ ist die Unter- 


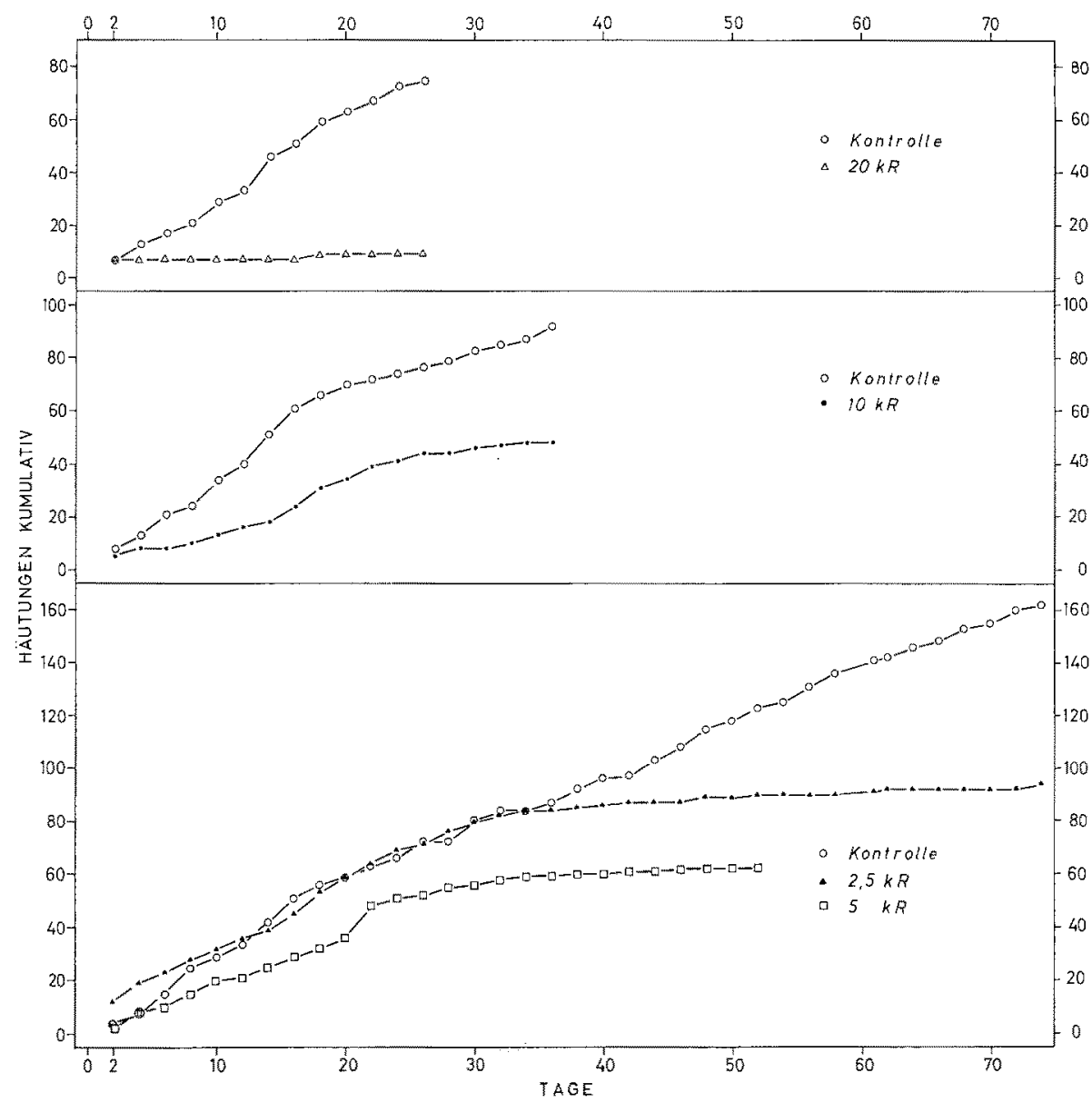

Abb. 25: Anzahl der Häutungen fortlaufend addiert bei $\delta \hat{\delta}$ von Gammarus duebeni nach einmaligen Röntgenbestrahlungen. Anzahl der Tiere pro Versuch: 100

drückung der Häutungen in diesem Zeitraum weniger stark. 28 Tage nach Versuchsbeginn sind in der Versuchs- und Kontrollgruppe etwa gleich viele Häutungen erfolgt, dann knickt die Häutungssummenkurve der Versuchstiere ab, und es erfolgen nur noch wenige Häutungen. Nach $2500 \mathrm{R}$ ist eine anfängliche Unterdrückung der Häutungen nicht mehr zu beobachten, aber auch hier wird die Häutungssummenkurve nach etwa 30 Tagen erheblich flacher. Das Abknicken der Häutungssummenkurven etwa 30 Tage nach der Bestrahlung kann seinen Grund nur darin haben, daß nach Bestrahlung mit letalen Dosen sich der Strahlenschaden zu diesem Zeitpunkt so weit entwickelt hat, daß Häutungen nicht oder nur schwer möglich sind. Die vielleicht geringe Anzahl der nach 30 Tagen noch am Leben befindlichen Tiere kann keine Rolle spielen, da nach Bestrahlungen mit $2500 \mathrm{R}$ zu diesem Zeitpunkt noch mehr als $50 \%$ der Versuchstiere am Leben sind, 


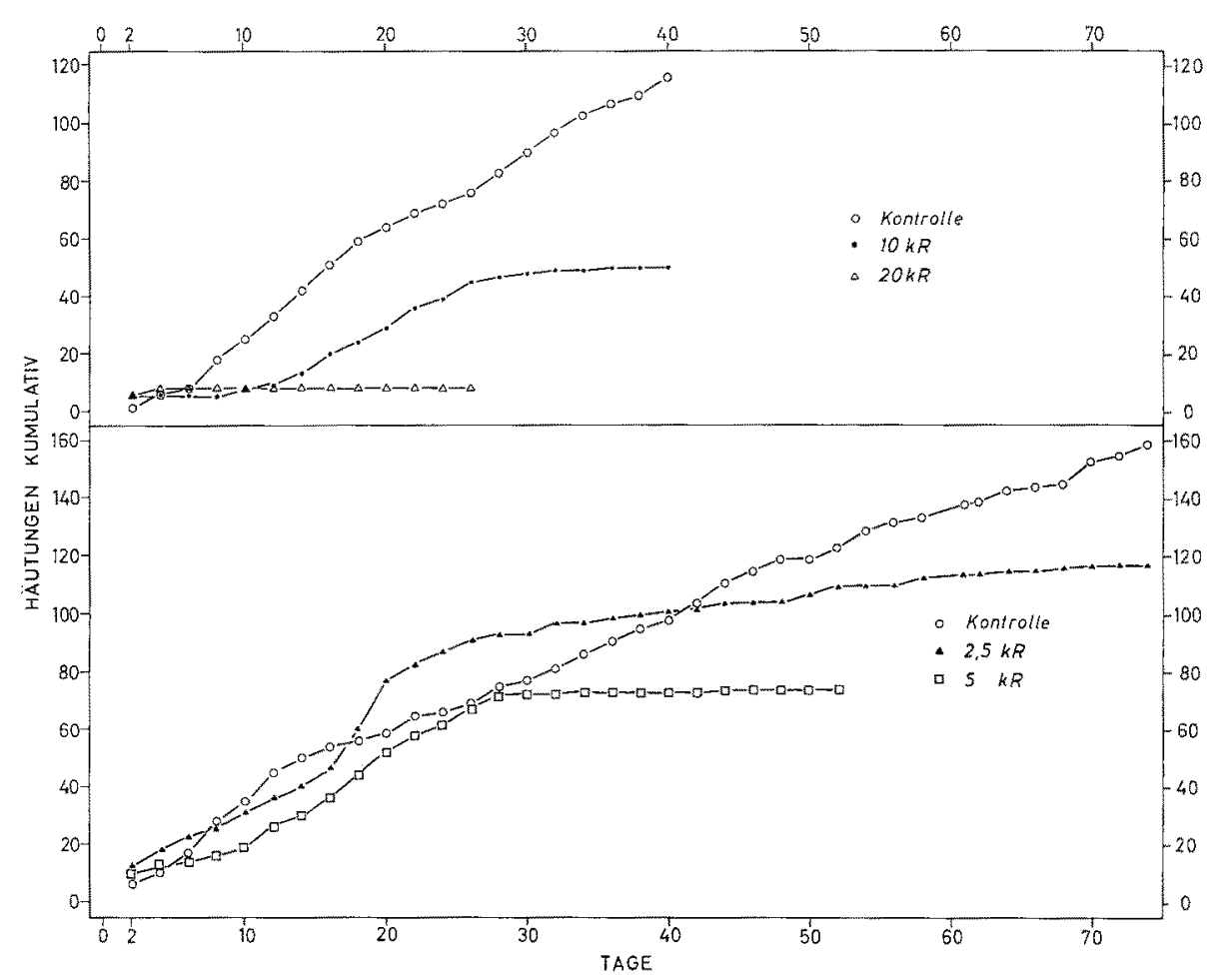

Abb. 26: Anzahl der Häutungen fortlaufend addiert bei $O Q$ von Gammarus duebeni nach einmaligen Röntgenbestrahlungen. Anzahl der Tiere pro Versuch: 100

Die größere Strahlenresistenz der ô đ̧ und 90 im ersten Quartal 1967 macht sich auch in einer geringeren Beeinflußbarkeit der Häutungsvorgänge bemerkbar. Die $\mathrm{Ab}$ bildungen 27 und 28 enthalten die Häutungssummenkurven der Versuche der Abbildungen 21 bis 24 mit Laboratoriumstieren und Tieren aus dem Freiland im ersten Quartal 1967. Die Laboratoriumstiere verhalten sich wie die Freilandtiere aus dem zweiten Quartal, deren Häutungssummenkurven die Abbildungen 25 und 26 zeigen. Bei den Tieren aus dem ersten Quartal 1967 sind nach Bestrahlungen mit $20000 \mathrm{R}$ noch zu einem späteren Zeitpunkt als bei den Laboratoriumstieren Häutungen möglich. Bei den ô $\delta$ sind nach der Bestrahlung mit $10000 \mathrm{R}$ nach 35 Tagen in der Versuchsgruppe genauso viele Häutungen erfolgt wie in der Kontrollgruppe. Aber auch hier knickt die Häutungssummenkurve der Versuchstiere $\mathrm{ab}$ und wird flacher.

Die Abbildung 29 enthält Häutungssummenkurven von jungen $q Q$, die kurz vor der Geschlechtsreife stehen oder gerade in sie eingetreten sind. Die Uberlebenskurven, die in der Abbildung $12 \mathrm{zu}$ finden sind, zeigen keinen signifikanten Unterschied. An den Häutungssummenkurven der Kontrolltiere ist deutlich zu erkennen, daß es sich um Tiere verschiedenen Alters handelt. Die Häutungsfrequenz bei den vor der Geschlechtsreife stehenden Tieren ist wesentlich höher und führt $\mathrm{zu} 203$ Häutungen innerhalb von 
36 Tagen, während bei den geschlechtsreifen jungen $ᄋ$ 우 im gleichen Zeitraum nur 126 Häutungen zu verzeichnen sind. Auch in diesen Versuchen ist bei den bestrahlten Tieren eine Unterdrückung der Häutungen zwischen dem 2. und 8. Tag nach der Bestrahlung zu beobachten. Die wenigen Tiere, die sich in diesem Zeitraum häuten, haben eine geringere Lebenserwartung als die Tiere, die sich unmittelbar davor oder danach häuten. Abbildung 30 zeigt für beide Versuche die mittleren Überlebenszeiten

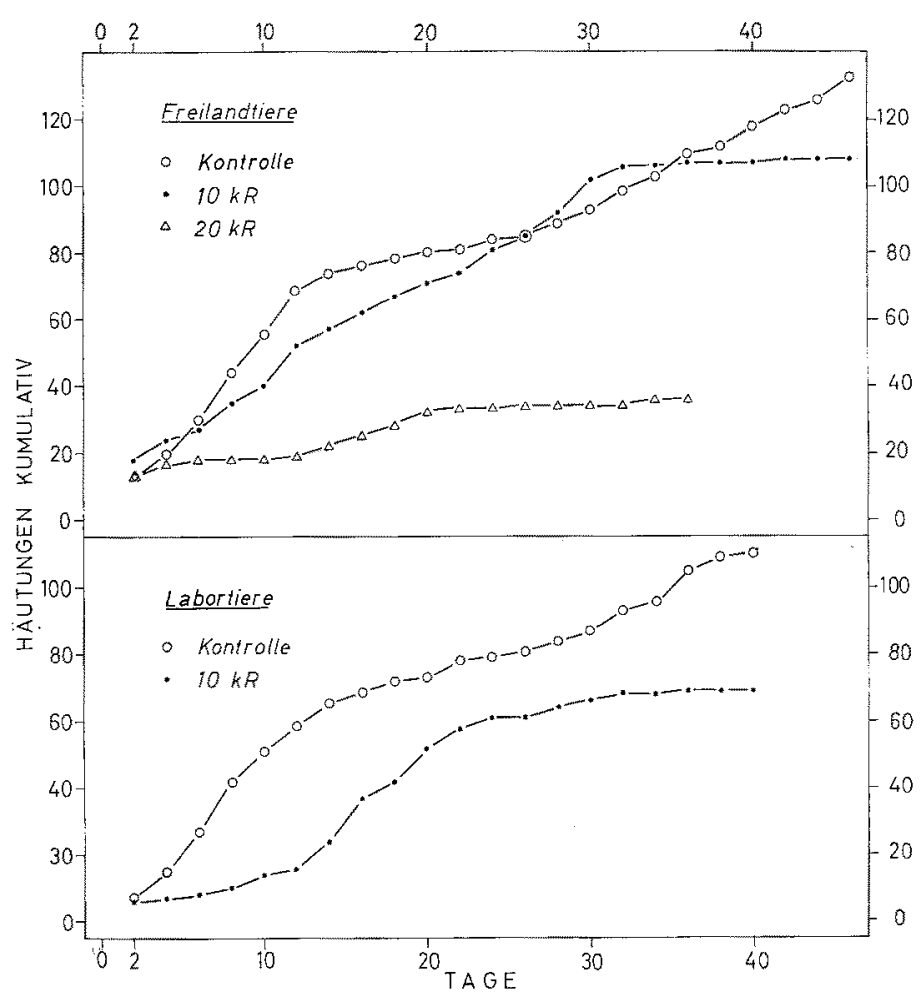

Abb. 27: Anzahl der Häutungen fortlaufend addiert bei $\hat{\partial} \delta$ von Gammarus duebeni aus dem Freiland im ersten Quartal des Jahres 1967 und aus Laboratoriumszuchten nach einmaligen Röntgenbestrahlungen. Anzahl der Tiere pro Versuch: 100

nach dem Tage der Häutung. Auch nach Bestrahlungen mit $5000 \mathrm{R}$ ist die Lebenserwartung der Tiere, die sich in dem kritischen Zeitraum häuten, verkürzt. Abbildung 31 gibt die mittleren Uberlebenszeiten von $\delta \delta$ und $q 9$ nach der Häutung nach einer Bestrahlung mit $5000 \mathrm{R}$ wieder. Die dazugehörigen Überlebenskurven finden sich in den Abbildungen 5 und 6, die Häutungssummenkurven in den Abbildungen 25 und 26.

Die Verkürzung der Lebenserwartung von Tieren, die sich in einem kritischen Zeitraum nach der Bestrahlung häuten, läßt vermuten, daß Versuchsgruppen, die sich zu verschiedenen Zeiten nach einer Bestrahlung häuten, unterschiedliche Überlebenszeiten haben. Diese Annahme wird gestützt durch die weitere Beobachtung, daß sowohl 
bei den Kontroll- als auch bei den Versuchstieren Todesfälle gehäuft am 4. Tag nach einer Häutung auftreten. Die Abbildungen 32 und 33 zeigen die Zahl der Todesfälle bei Kontroll- und Versuchstieren zu verschiedenen Zeiten nach der letzten Häutung

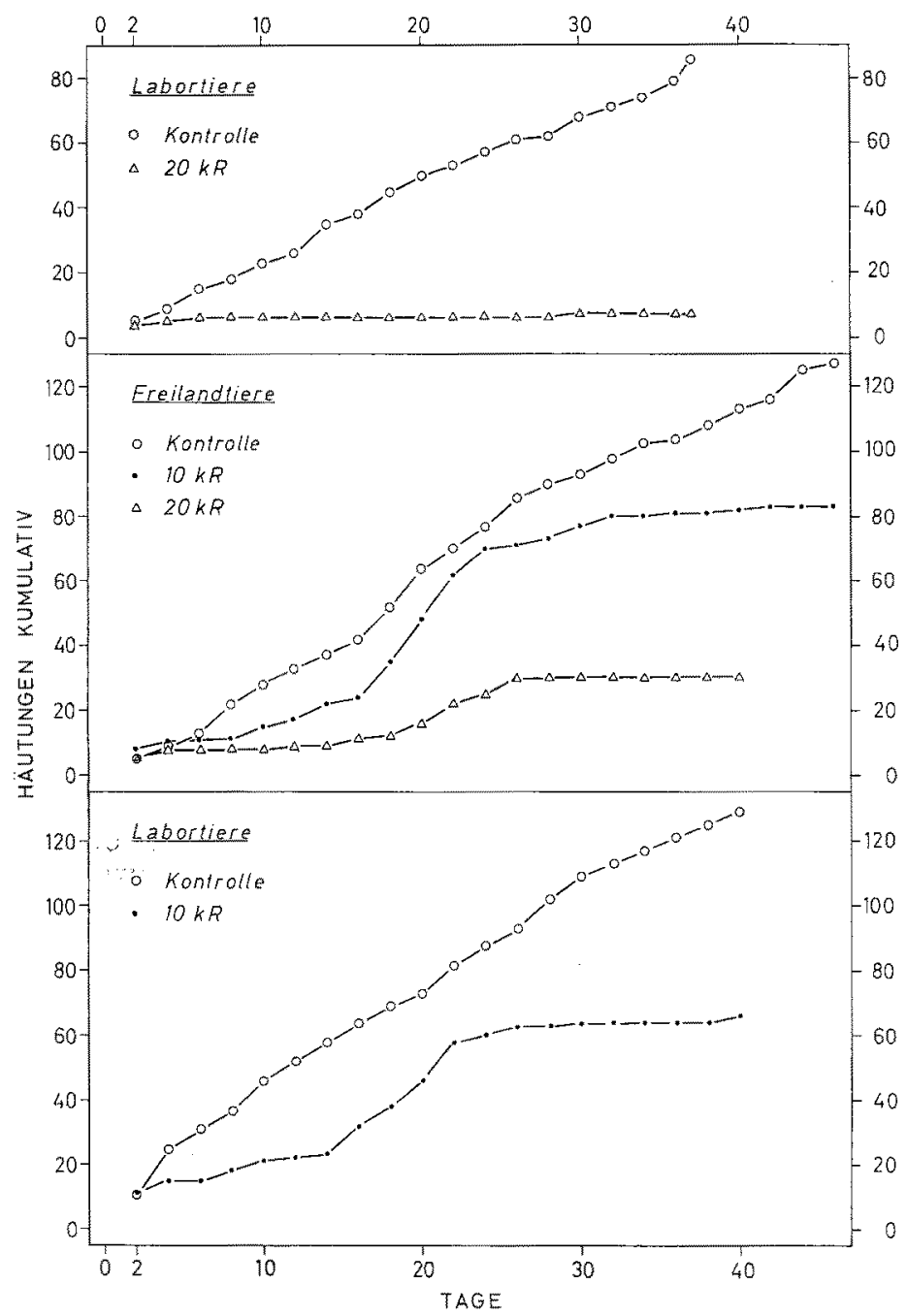

Abb. 28: Anzahl der Häutungen fortlaufend addiert bei $Q Q$ von Gammarus duebeni aus dem Freiland im ersten Quartal des Jahres 1967 und aus Laboratoriumszuchten nach einmaligen Röntgenbestrahlungen. Anzahl der Tiere pro Versuch: 100

vor dem Tode. Alle Kurven sind einmal geglättet worden. Bei den Kontrolltieren (Abb. 32) treten 3 Gipfel auf: der erste 4 Tage, der zweite etwa 26 Tage nach einer Häutung. Die beiden Gipfel liegen 22 Tage auseinander, was der Länge des Häutungsintervalls bei den $ㅇ$ entspricht. Von den Tieren, die 26 Tage nach einer Häutung sterben, kann angenommen werden, daß sie die Häutung, die nach 22 Tagen erfolgen 
müßte, nicht mehr durchführen können und 4 Tage nach dem fälligen Häutungstermin sterben. Unklar ist, was um den 40. Tag herum geschieht, an dem der dritte Gipfel auftritt. Es könnte sich um Tiere handeln, die gleichfalls die nach 22 Tagen fällige

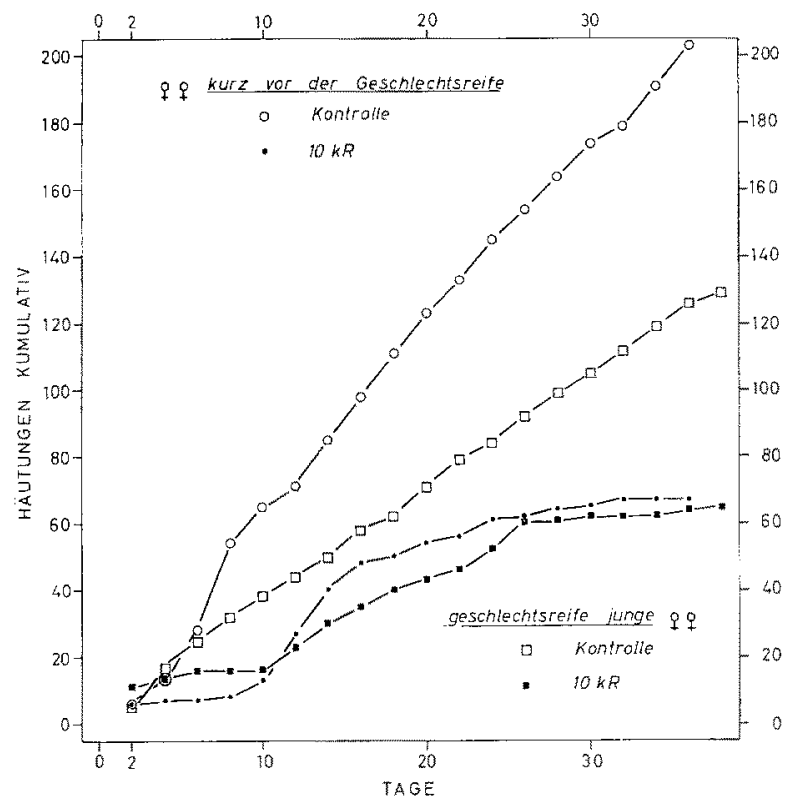

Abb. 29: Anzahl der Häutungen fortlaufend addiert bei jungen 9 우 von Gammarus duebeni nach einmaliger Röntgenbestrahlung mit $10 \mathrm{kR}$. Anzahl der Tiere pro Versuch: 100 (aus Laboratoriumszuchten)

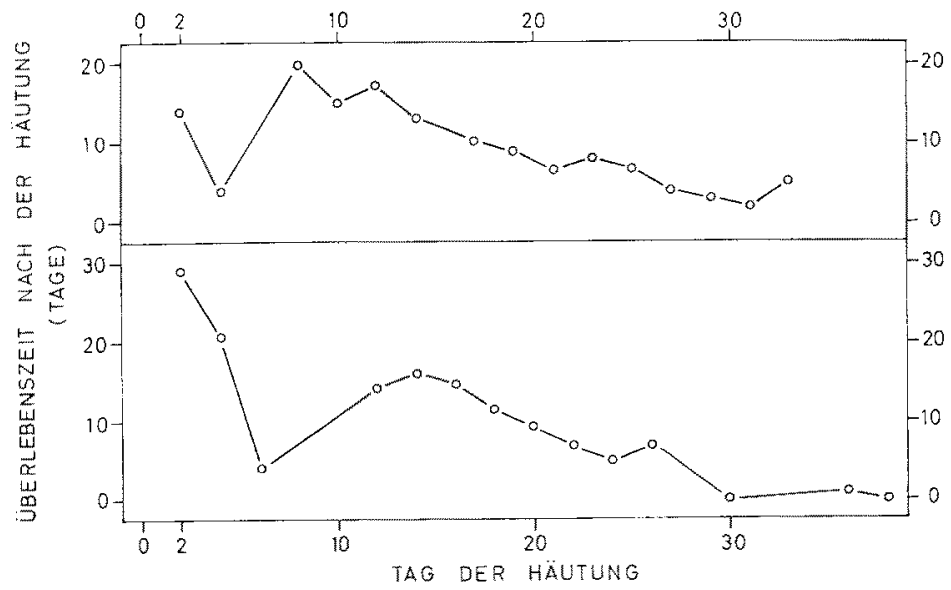

Abb. 30: Mittlere Uberlebenszeit nach der Häutung in Abhängigkeit vom Zeitpunkt der Häutung bei jungen $O Q$ von Gammarus duebeni aus Laboratoriumszuchten. Oberer Teil der Abbildung: Tiere kurz vor der Geschlechtsreife; unterer Teil: junge geschlechtsreife Tiere. Bestrahlungsdosis: $10 \mathrm{kR}$ 
Häutung nicht durchführen, nun aber nicht 4 Tage nach der fälligen Häutung sterben, sondern weiter überleben und vor dem Eintritt in die nächste Häutung sterben. Später wird gezeigt werden, daß es nicht nur nach, sondern auch vor der Häutung eine kritische Phase gibt, in der die Tiere sterben können. Bereits die Abbildung 33 weist auf eine solche Phase hin. Bei den mit $10000 \mathrm{R}$ bestrahlten $q \circ$ tritt 20 Tage nach der letzten Häutung vor dem Tode ein zweiter Gipfel auf, der 2 Tage vor der nächsten zu

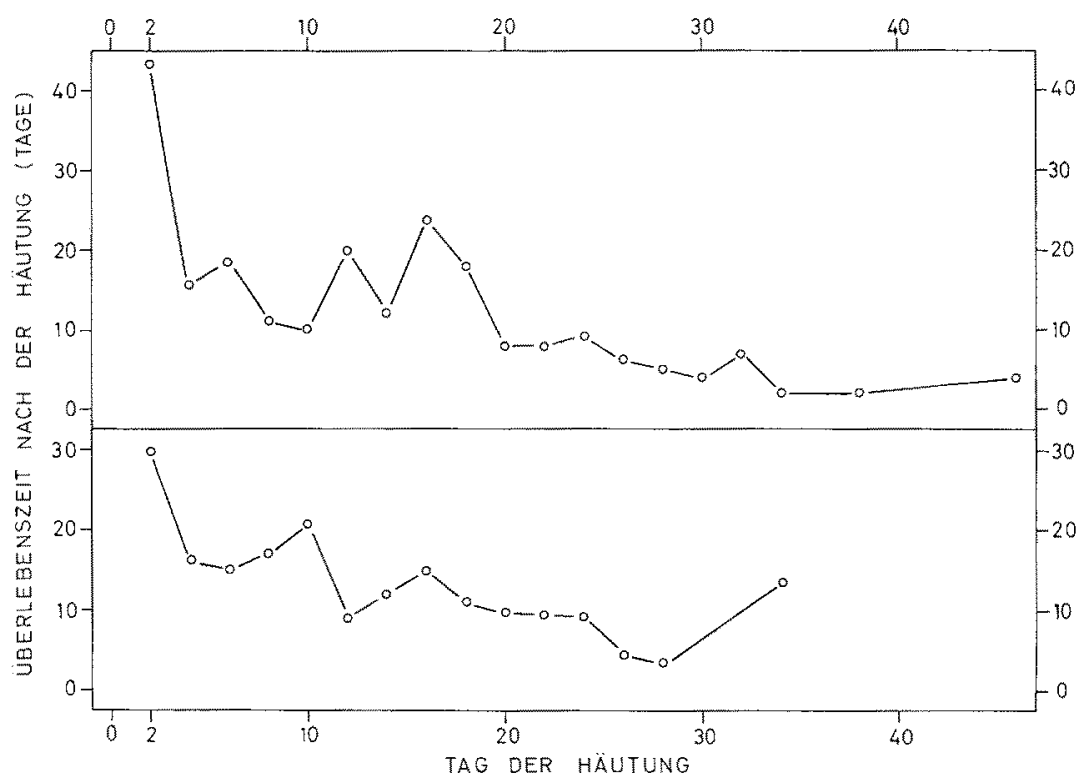

Abb. 31: Mittlere Uberlebenszeit nach der Häutung in Abhängigkeit vom Zeitpunkt der Häutung bei $\hat{\partial} \hat{\partial}$ und $Q \mathcal{Q}$ von Gammarus duebeni. Oberer Teil der Abbildung: $\hat{\partial} \hat{\partial}$; unterer Teil: $O Q$. Bestrahlungsdosis: $5 \mathrm{kR}$

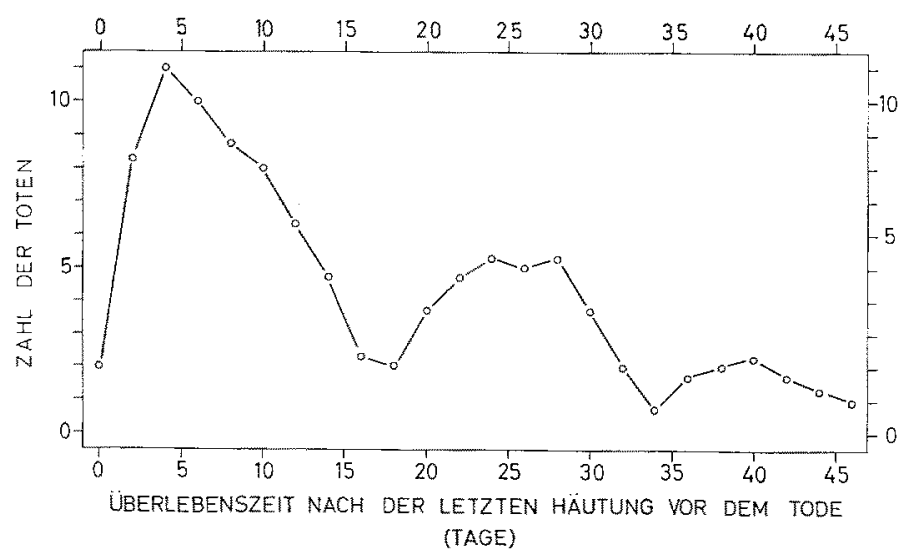

Abb. 32: Zahl der Todesfälle bei Kontrolltieren zu verschiedenen Zeiten nach der letzten Häutung vor dem Tode. 107 오 von Gammarus duebeni 


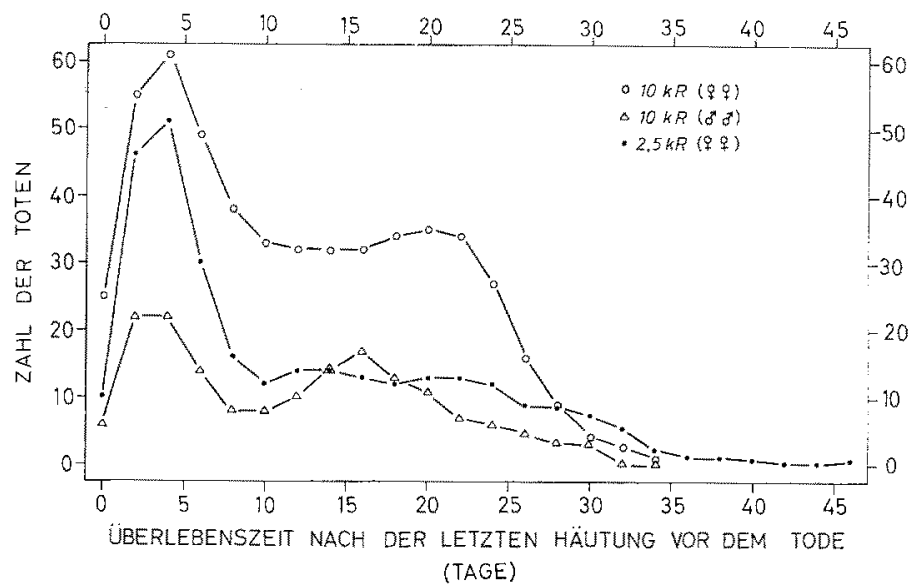

Abb. 33: Zahl der Todesfälle bei of of und $Q 9$ von Gammarus duebeni zu verschiedenen Zeiten nach der letzten Häutung vor dem Tode. $ᄋ$ 우 $(2,5 \mathrm{kR}): 313$ Tiere; 9 우 $(10 \mathrm{kR}): 534$ Tiere; Oे $\mathrm{O}^{\pi}(10 \mathrm{kR}): 180$ Tiere

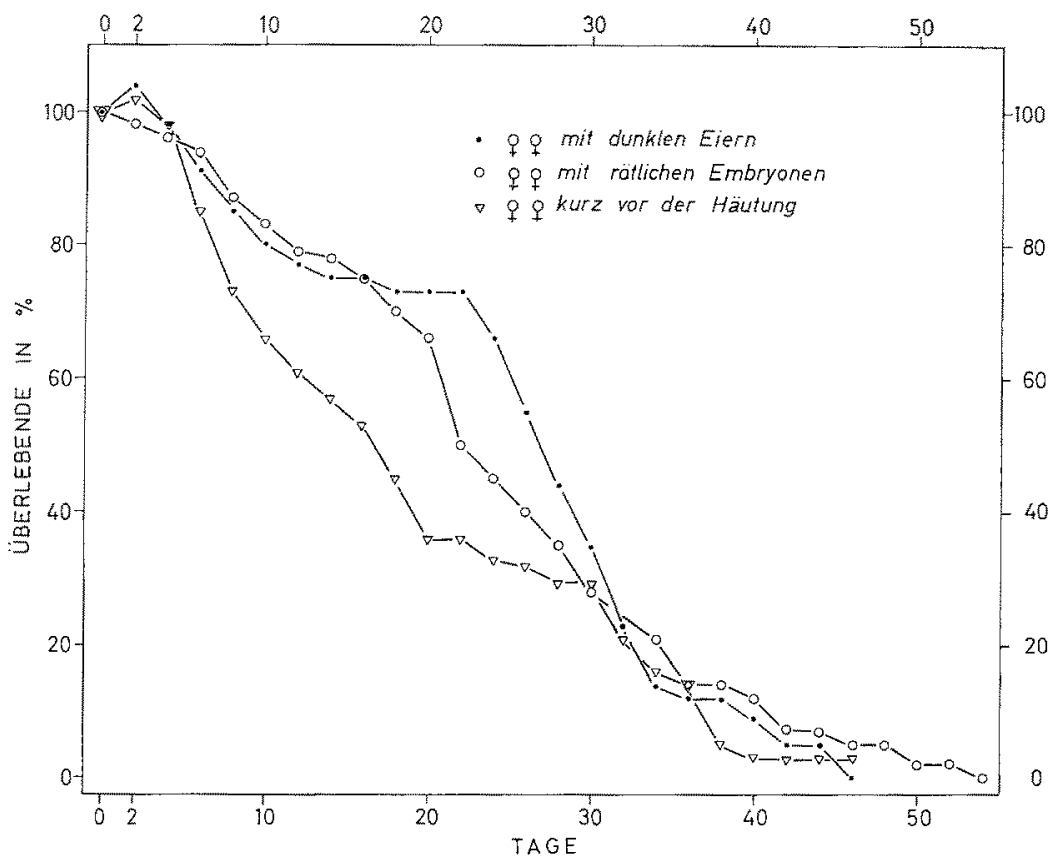

Abb. 34: Überlebenskurven dreier Gruppen von $q Q$ von Gammarus duebeni nach einmaliger Röntgenbestrahlung mit $10 \mathrm{kR}$. Die Gruppen häuten sich zu verschiedenen Zeiten nach der Bestrahlung. Angaben in Prozent der Zahl der jeweils lebenden Kontrolltiere. Anzahl der Tiere pro Versuch: 50 (aus Laboratoriumszuchten)

erwartenden Häutung liegt. Bei den $\hat{\delta} \hat{\delta}$ erfolgen die Häutungen in Abständen von 34 bis 42 Tagen. Der zweite Gipfel erscheint bei ihnen nach einer Bestrahlung mit $10000 \mathrm{R}$ nach 16 Tagen. Eine Erklärung für die Häufung der Todesfälle 16 Tage nach einer Häutung bei den $\partial \partial \mathrm{kann}$ nicht gegeben werden. Es ist in allen dargestell- 
ten Fällen damit zu rechnen, daß bei Tieren, die eine längere Überlebenszeit nach der letzten Häutung vor dem Tode aufweisen, als es der Länge des Häutungsintervalls entspricht, Häutungen nicht registriert wurden, weil die Tiere ihre Exuvien entweder total oder so weit aufgefressen hatten, daß die Reste übersehen wurden. Zur Aufstellung der Kurve der Abbildung 32 wurden die Kontrollen aller mit 2500 und $5000 \mathrm{R}$ bestrahlten Gruppen benutzt. Da die meisten Kontrolltiere das Ende der Versuche überlebten, wurden nur 107 Tiere gezählt, die sich während der Versuchszeit vor dem Tode gehäutet hatten.

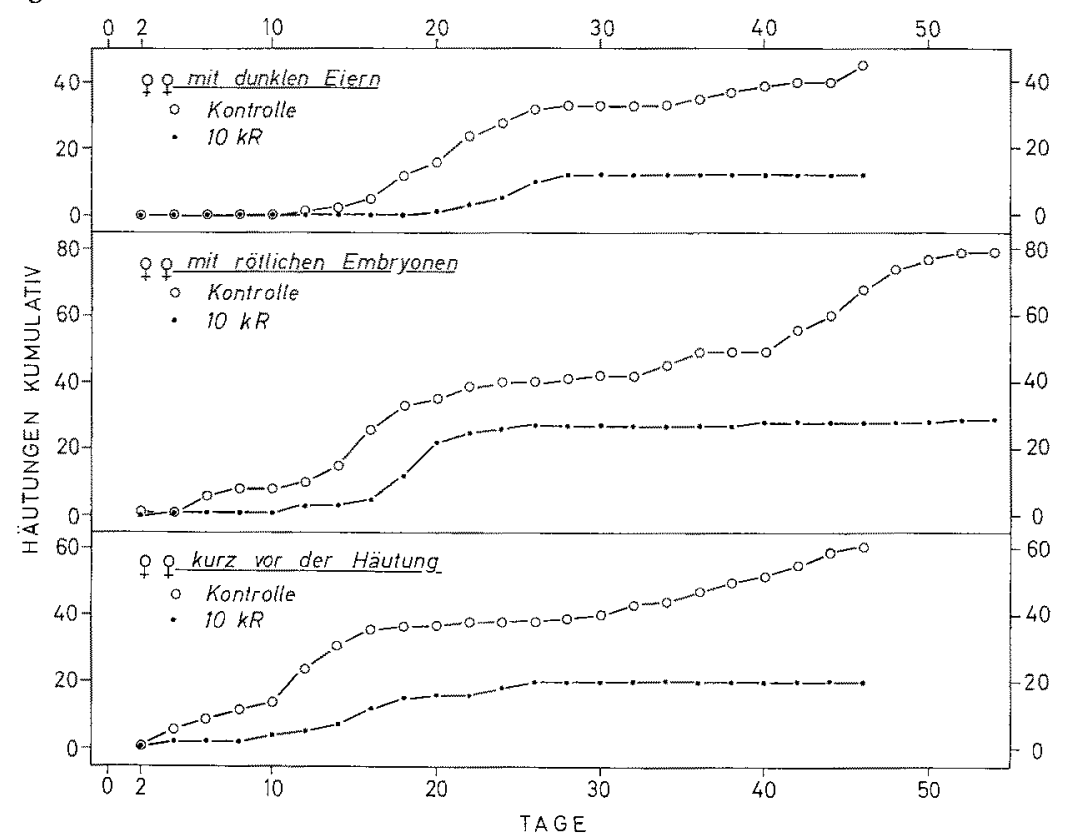

Abb. 35: Anzahl der Häurungen fortlaufend addiert bei $q Q$ von Gammarus duebeni. Drei Gruppen von je 50 Versuchs- und Kontrolltieren sind so ausgewählt worden, daß die Häutungen zu verschiedenen Zeiten nach der Bestrahlung erfolgen

Um den vermuteten Zusammenhang zwischen dem Termin der Häutung und der mittleren Überlebenszeit aufzudecken, wurden Versuche mit Tieren durchgeführt, die sich synchron zu verschiedenen Zeiten nach einer Bestrahlung häuteten. Es wurden $q$ q benutzt, weil bei ihnen durch das Zusammenfallen von Häutung und Oviposition der Zeitpunkt der nächsten Häutung durch den Reifezustand der Embryonen im Marsupium bestimmt werden kann. Wir wählten Tiere, die zum Zeitpunkt der Bestrahlung dunkle Eier, rötliche oder fast reife Embryonen trugen. Unmittelbar nach der Oviposition sind die Eier durch den Dotter dunkel gefärbt, 5 bis 11 Tage später, nachdem die Vorgänge der Furchung beendet sind bzw. die der Organbildung eingesetzt haben, sind im Brutraum erst rötliche und dann rötlich-gelbe Embryonen zu finden.

Abbildung 34 zeigt die Uberlebenskurven dreier Versuchsgruppen von $ᄋ$ 우 von Gammarus duebeni, die zum Zeitpunkt der Bestrahlung dunkle Eier, rötliche bzw. fast reife Embryonen im Marsupium trugen. Die mittleren Oberlebenszeiten sind deutlich verschieden; je später im Häutungsintervall die Bestrahlung erfolgt, desto kürzer 


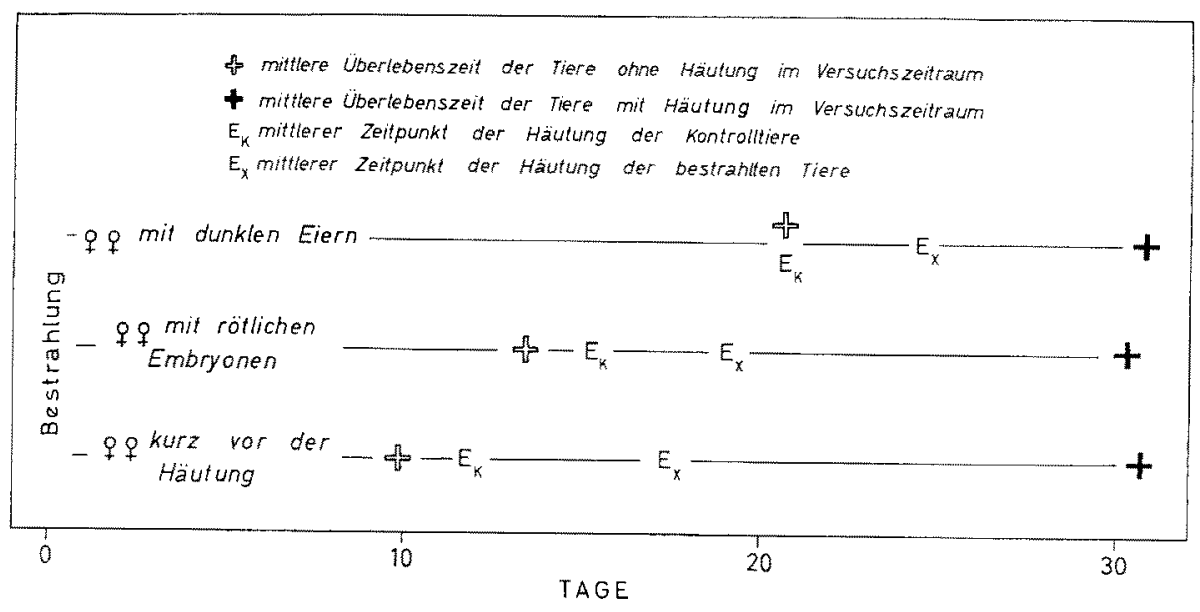

Abb. 36: Mittlere Uberlebenszeiten und mittlere Zeitpunkte der Häutungen bei $Q Q$ von Gammarus duebeni. Drei Gruppen von je 50 Versudss- und Kontrolltieren. Die Gruppen häuten sich zu verschiedenen Zeitpunkten nach der Bestrahlung. Bestrahlungsdosis: $10 \mathrm{kR}$

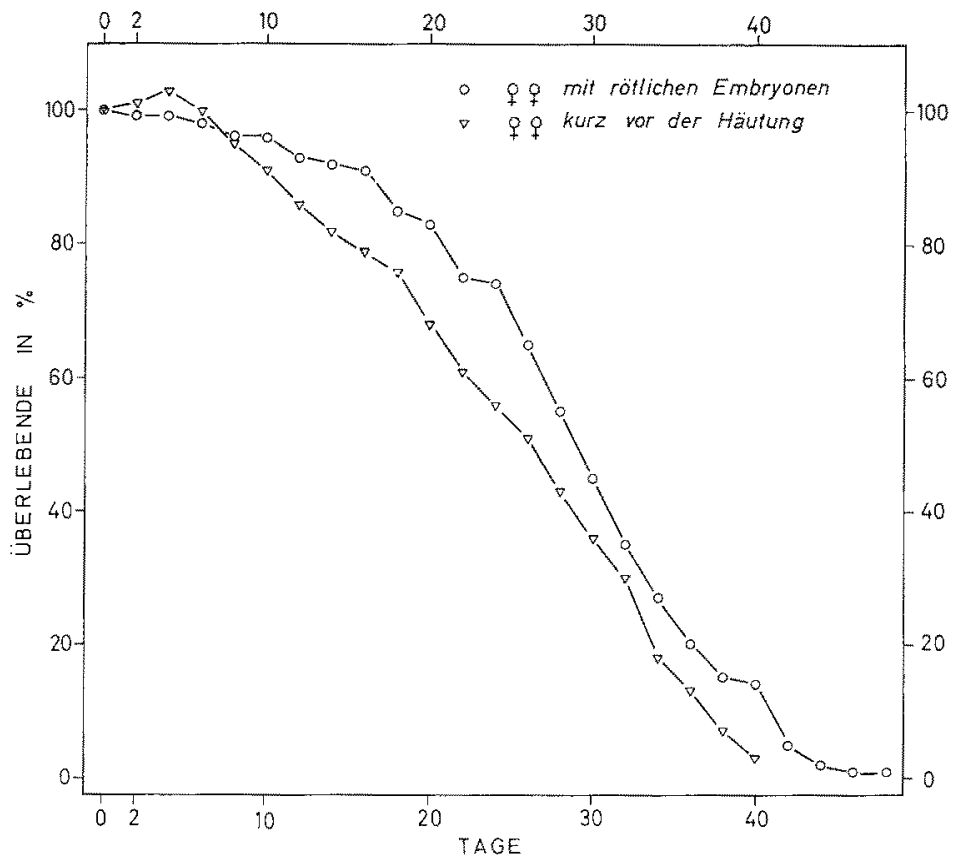

Abb. 37: Oberlebenskurven zweier Gruppen von Gammarus duebeni $Q Q$ nach einmaliger Röntgenbestrahlung mit $10 \mathrm{kR}$ im ersten Quartal 1967. Die Gruppen häuten sich zu verschiedenen Zeiten nach der Bestrahlung. Angaben in Prozent der Zahl der jeweils lebenden Kontrolltiere. Anzahl der Tiere pro Versuch: 100

ist die mittlere Uberlebenszeit. Der Unterschied zwischen den Überlebenskurven von Tieren mit dunklen Eiern und Tieren mit rötlichen Embryonen im Brutsack ist mit $0,1 \%$ gesichert. Die Versuche wurden mit je 50 aus Laboratoriumszuchten 


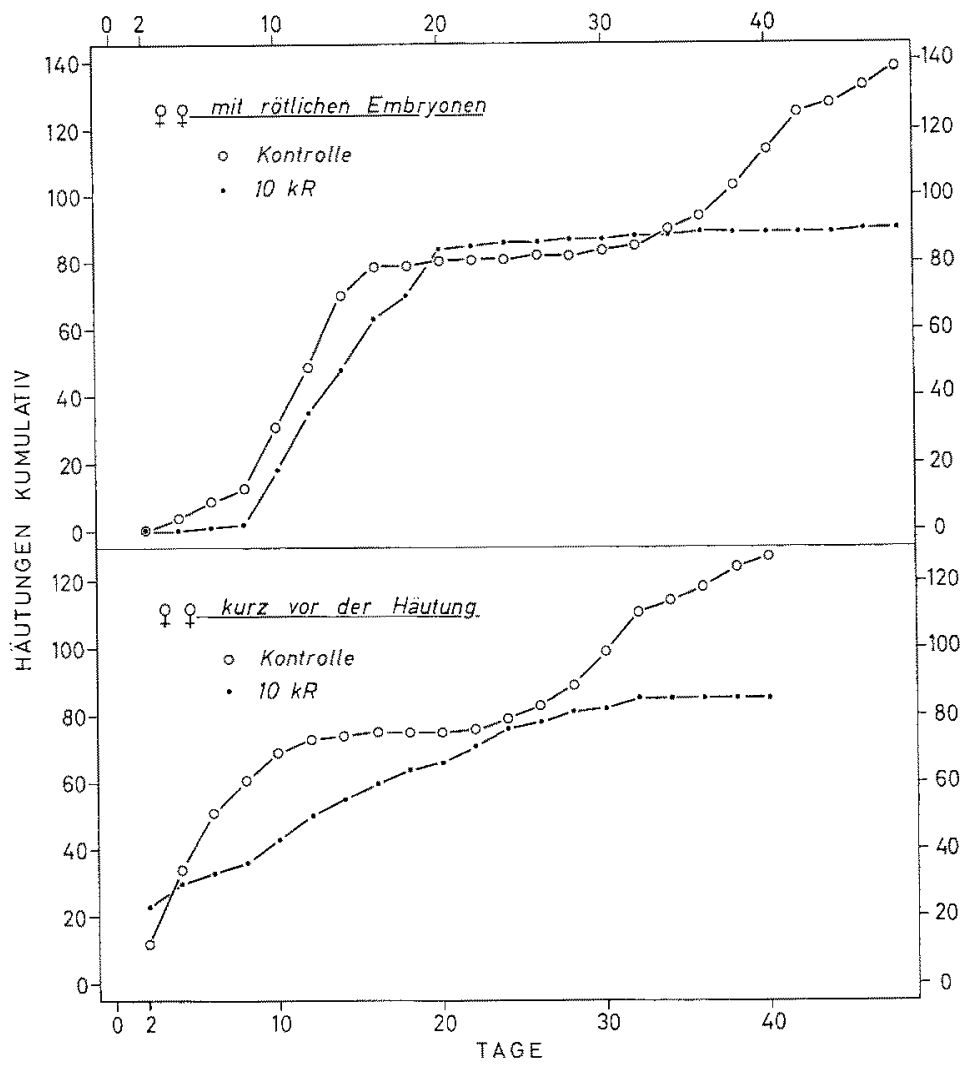

Abb. 38: Anzahl der Häutungen fortlaufend addiert bei $ᄋ$ 우 von Gammarus duebeni im ersten Quartal 1967. Zwei Gruppen von je 100 Versuchs- und Kontrolltieren sind so ausgewählt worden, daß die Häutungen zu verschiedenen Zeiten nach der Bestrahlung erfolgen

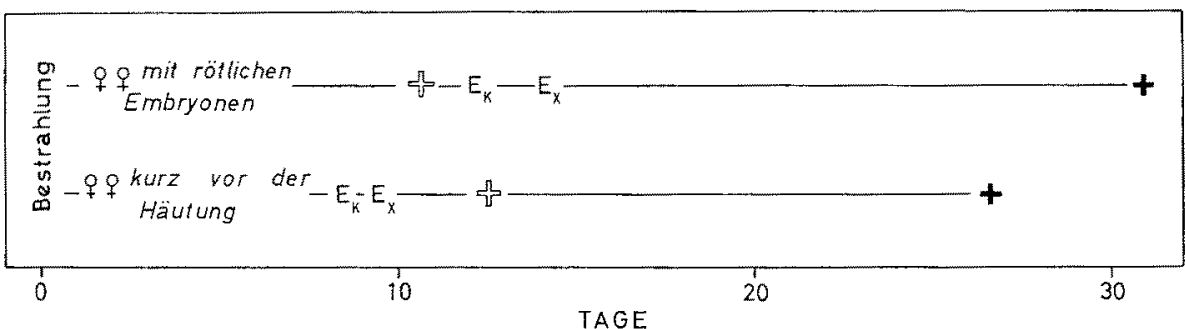

Abb. 39: Mittlere Oberlebenszeiten und mittlere Zeitpunkte der Häutungen bei zwei Gruppen von je 100 Versuchs- und Kontrolltieren von Gammarus duebeni Oᄋ. Erstes Quartal 1967. Die Gruppen häuten sich zu verschiedenen Zeitpunkten nach der Bestrahlung. Bestrahlungsdosis: $10 \mathrm{kR}$. (Symbole siehe Abb. 36)

entnommenen Tieren durchgeführt. Es tritt eine stark bimodale Verteilung der Todesfälle auf. Die Häutungssummenkurven der Versuchs- und Kontrollgruppen enthält die Abbildung 35. Die Häutungen erfolgen bei den bestrahlten Gruppen später als bei den 
Kontrolltieren. Frühere Versuche zeigten eine Unterdrückung der Häutung zwischen dem 2. und 8. Tag nach der Bestrahlung. Offensichtlich handelte es sich dort weniger um eine Unterdrückung der Häutung in einem bestimmten Zeitraum nach der Bestrahlung, sondern um eine generelle Verzögerung der Häutung bei bestrahlten Tieren, die auch dann noch eintreten kann, wenn die Häutungen erst 10 Tage nach einer Bestrahlung erfolgen.

In jeder Versuchsgruppe treten Tiere auf, die sich im Versuchszeitraum vor ihrem Tode häuten oder sterben, ohne sich vorher im Versuchszeitraum gehäutet zu haben.

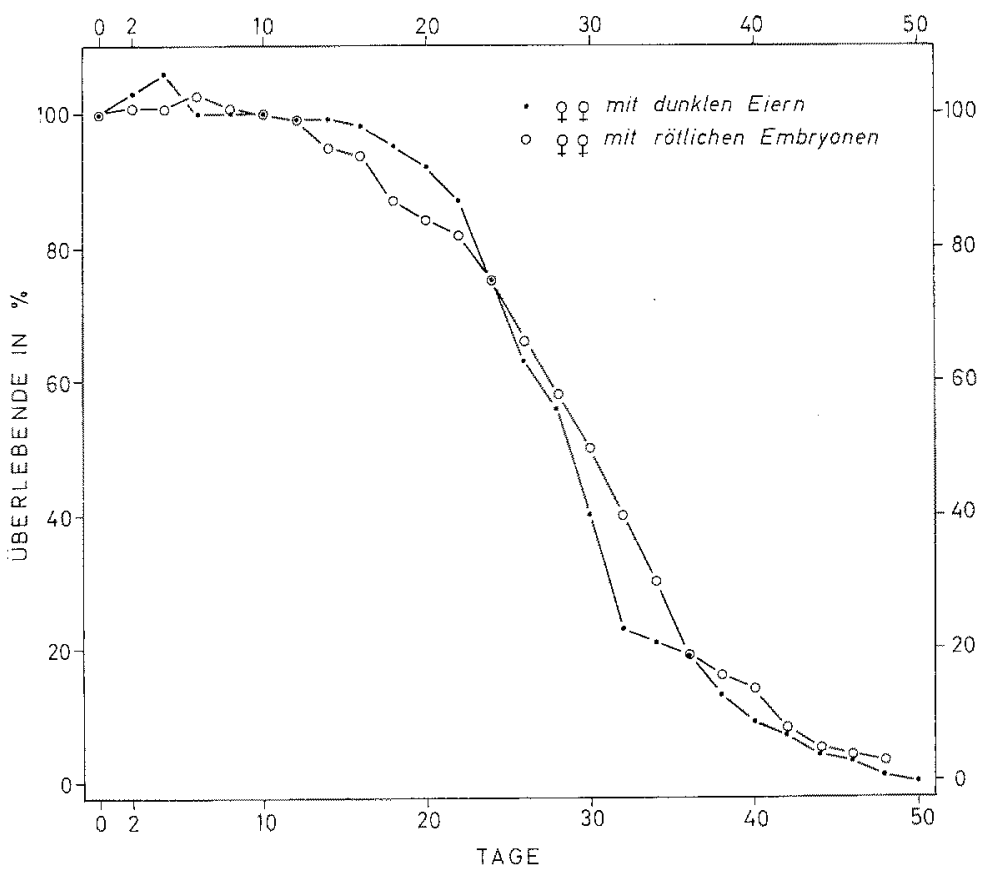

Abb. 40: Uberlebenskurven zweier Gruppen von Gammarus duebeni $P Q$ nach einmaliger Röntgenbestrahlung mit $5 \mathrm{kR}$. Die Gruppen häuten sich zu verschiedenen Zeiten nach der Bestrahlung. Angaben in Prozent der Zahl der jeweils lebenden Kontrolltiere. Anzahl der Tiere pro Versuch: 100 (aus Laboratoriumszuchten)

Die Abbildung 36 enthält Angaben iber die mittleren Öberlebenszeiten der Tiere mit und ohne Häutung im Versuchszeitraum. Außerdem sind die mittleren Zeitpunkte der Häutungen bei den Kontroll- und Versuchsgruppen angegeben werden. Es besteht ein Zusammenhang zwischen dem Zeitpunkt des Todes der im Versuchszeitraum sich nicht häutenden Tiere und dem Zeitpunkt der Häutung bei den übrigen Tieren. Je später die Häutung nach der Bestrahlung erfolgt, desto später erfolgt auch der Tod der sich im Versuchszeitraum nicht häutenden Tiere. Diese sterben $\mathrm{im}$ Durchschnitt 4 bis 7 Tage vor dem Zeitpunkt, an dem eine Häutung erfolgen müßte. Die Tiere mit Häutung im Versuchszeitraum haben in allen drei Versuchen die gleiche mittlere Uberlebenszeit. $\mathrm{Da}$ - wie die starke Bimodalität der Uberlebenskurven zeigt - die Zahl der Tiere, die 
ohne im Versuchszeitraum erfolgende Häutung sterben, sehr hoch ist, wird durch sie die mittlere Uberlebenszeit der Versuchsgruppen wesentlich beeinflußt.

In einem weiteren Versuch wurden 99 von Gammarus duebeni in Frühjahr 1967 mit $10000 \mathrm{R}$ bestrahlt. Es handelt sich um zwei Gruppen mit rötlichen bzw. fast reifen Embryonen im Marsupium. Der Unterschied zwischen den Oberlebenskurven, den die Abbildung 37 zeigt, ist nicht signifikant. Die Zahl der Tiere, die starben, ohne sich vorher im Versudhszeitraum zu häuten, war gering. Die Häutungssummenkurven enthält die Abbildung 38. Auch hier ist eine Verzögerung des Eintritts der Häutungen bei den Versuchsgruppen zu beobachten. In den Versuchen wurden je 100 Kontroll-

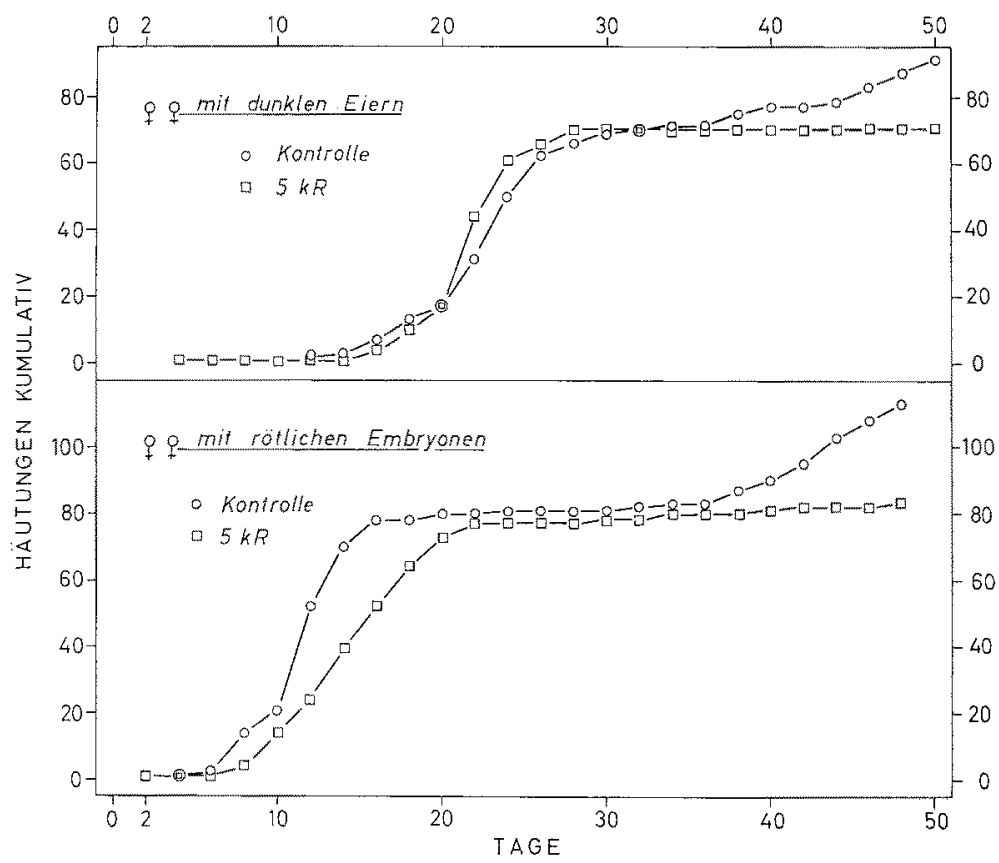

Abb. 41: Anzahl der Häutungen fortlaufend addiert bei $Q 9$ von Gammarus duebeni. Zwei Gruppen von je 100 Versuchs- und Kontrolltieren sind so ausgewählt worden, daß die Häutungen $z u$ verschiedenen Zeiten nach der Bestrahlung erfolgen

und Versuchstiere benutzt. Der Unterschied zwischen den mittleren Uberlebenszeiten (Abb. 39) entsteht allein durch die Tiere, die sich im Versuchszeitraum häuten und im Durchschnitt etwa 17 Tage nach der Häutung sterben.

Zwei Gruppen von 9 우 von Gammarus duebeni mit dunklen Ejern bzw. rötlichen Embryonen im Brutsack wurden mit $5000 \mathrm{R}$ bestrahlt. Die Tiere entstammen Laboratoriumszuchten und zeigen keinen Unterschied in den Uberlebenskurven (Abb. 40). Die Häutung setzt nur bei den sich früher häutenden $q$ m mit rötlichen Embryonen verspätet ein (Abb. 41). Die Zahl der Tiere, die starben, ohne sich vorher im Versuchszeitraum zu häuten, war gering. Da die Häutungen der $q 9$ mit dunklen Eiern zu einem relativ späten Zeitpunkt nach der Bestrahlung erfolgten, ist der Abstand zwischen Häutung und Phase akuter Sterblichkeit bei ihnen so gering, daß ein Unterschied 


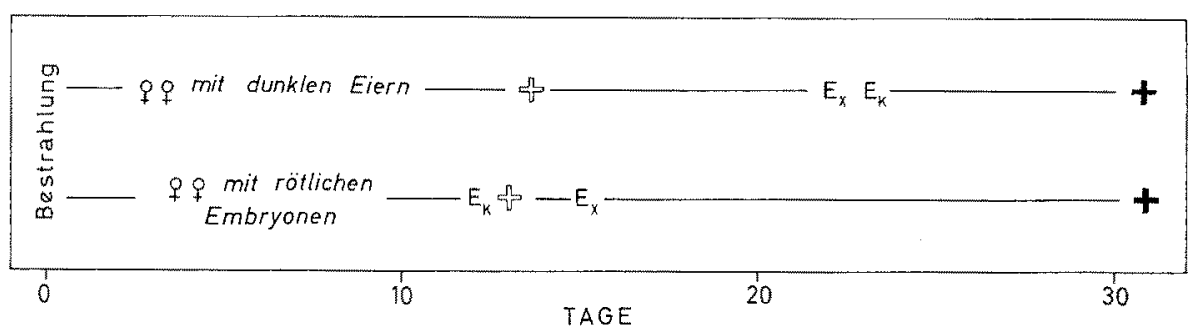

Abb. 42: Mittlere Überlebenszeiten und mittlere Zeitpunkte der Häutungen bei zwei Gruppen von je 100 Versuchs- und Kontrolltieren von Gammarus duebeni $\$ 9$. Gruppen hảuten sich zu verschiedenen Zeitpunkten nach der Bestrahlung. Bestrahlungsdosis: $5 \mathrm{kR}$.

(Symbole siehe Abb. 36)

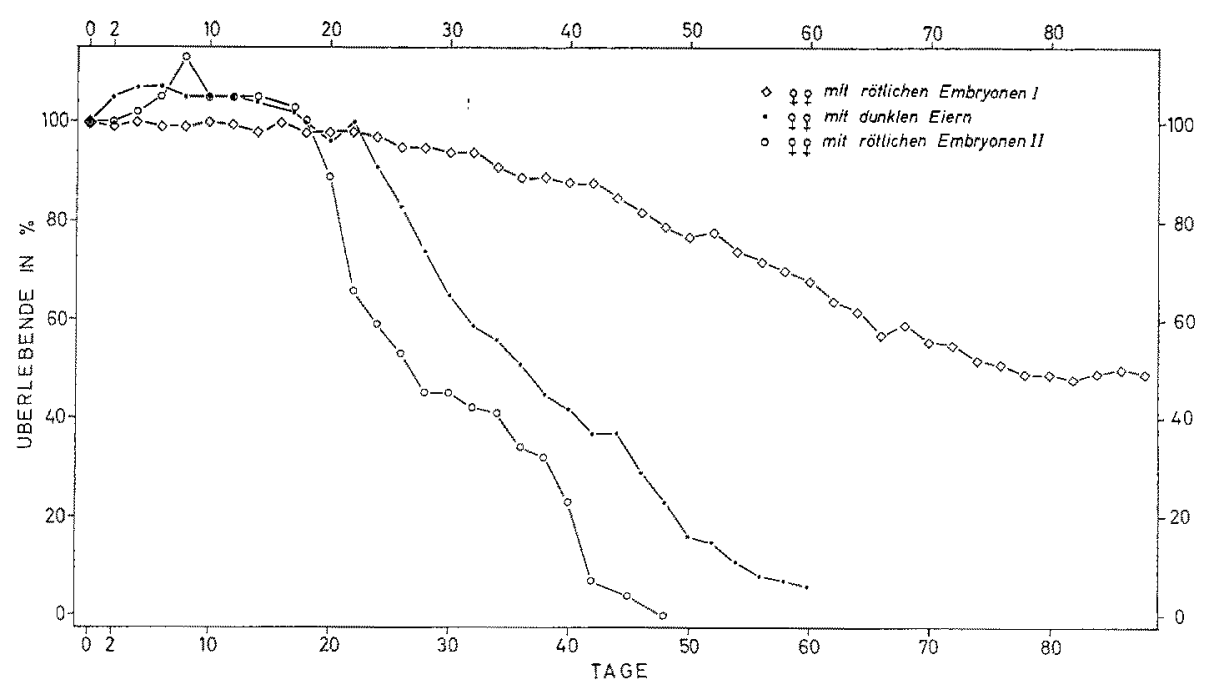

Abb. 43: Uberlebenskurven dreier Gruppen von Gammarus duebeni 9 nach einmaliger Röntgenbestrahlung mit 2,5 kR. Angaben in Prozent der Zahl der jeweils lebenden Kontrolltiere. Anzahl der Tiere mit rötlichen Embryonen I im ersten Quartal 1967: 100; mit dunklen Eiern (aus Laboratoriumszuchten): 100; mit rötlichen Embryonen II (aus Laboratoriumszuchten): 50

zwischen den mittleren Uberlebenszeiten nicht auftreten kann. Die Tiere sterben daher trotz unterschiedlicher Häutungszeitpunkte im Mittel zur gleichen Zeit (Abb. 42).

In Abbildung 43 sind Überlebenskurven von weiblichen Gammarus duebeni dargestellt, die mit $2500 \mathrm{R}$ bestrahlt wurden und zum Zeitpunkt der Bestrahlung dunkle Eier oder rötliche Embryonen im Marsupium trugen. In die Abbildung eingetragen wurde ferner die Uberlebenskurve einer Gruppe von 90 , die im ersten Quartal 1967 bestrahlt wurde. Es soll an diesem Beispiel nur noch einmal die höhere Strahlenresistenz der Tiere in der Zeit von Januar bis März 1967 demonstriert werden. Der Unterschied zwischen den Uberlebenskurven der hier interessierenden und aus Laboratoriumszuchten stammenden Gruppen ist mit 0,1\% Irrtumswahrscheinlichkeit ge- 


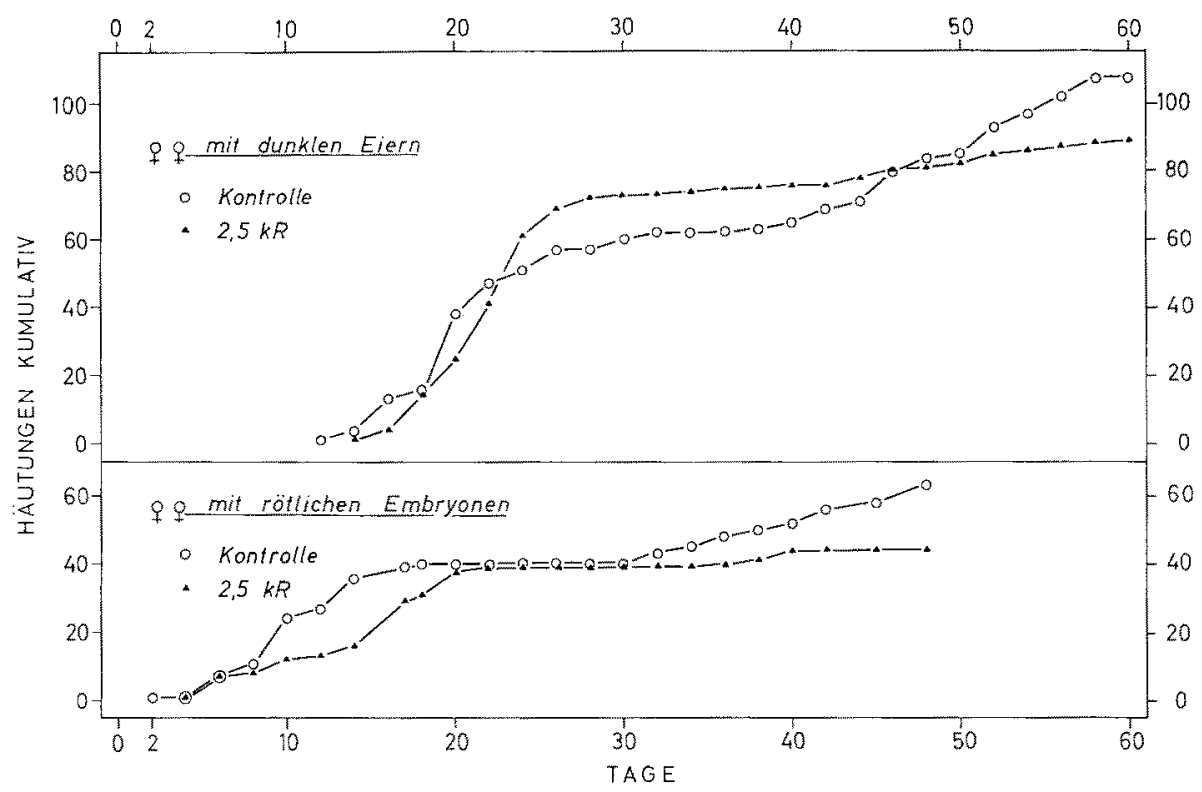

Abb. 44: Anzahl der Häutungen fortlaufend addiere bei 99 von Gammarus duebeni. Zwei Gruppen von Versuchs- und Kontrolltieren sind so ausgewählt worden, daß die Häutungen zu verschiedenen Zeiten nach der Bestrahlung erfolgen. Anzahl der $\nmid \uparrow$ mit dunklen Eiern: 100; Anzahl der $\$$ ㅇ mit rötlichen Embryonen: 50

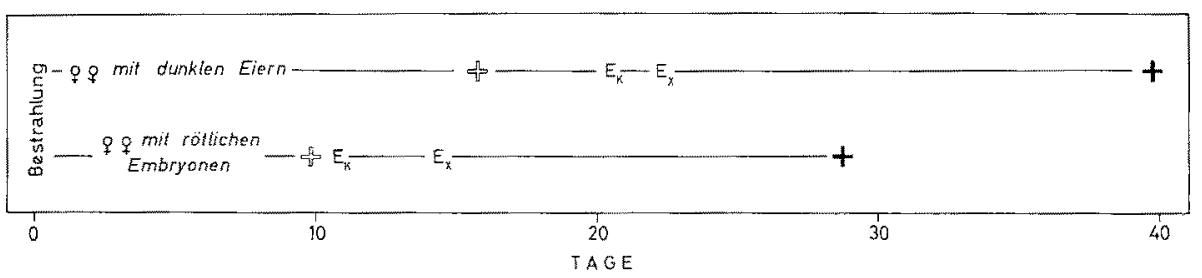

Abb. 45: Mittlere Überlebenszeiten und mittlere Zeitpunkte der Häutungen bei zwei Gruppen von Versuchs- und Kontrolltieren von Gammarus duebeni 웅. Die Gruppen häuten sich zu verschiedenen Zeitpunkten nach der Bestrahlung. Bestrahlungsdosis: 2,5 kR. Anzahl der $O O$ mit dunklen Eiern: 100; Anzahl der Q 9 mit rötlichen Embryonen: 50. (Symbole siehe Abb. 36)

sichert. Die Häutungssummenkurven zeigt Abbildung 44. Der Versuch mit 90 mit dunklen Eiern wurde mit 100 Kontroll- und Versuchstieren durchgeführt, der Versuch mit 99 mit rötlichen Embryonen mit nur 50 Kontroll- und Versuchstieren. Bei den sich bald nach der Bestrahlung häutenden Tieren mit rötlichen Embryonen im Brutsack ist eine Verzögerung des Eintritts der Häutungen zu beobachten. Da nach Bestrahlungen mit $2500 \mathrm{R}$ die Phase der akuten Sterblichkeit später einsetzt als nach Bestrahlungen mit höheren Dosen, kann ein Unterschied in den mittleren Öberlebenszeiten in Abhängigkeit vom Zeitpunkt der Häutung auftreten (Abb. 45). 


\section{SCHLUSSBETRACHTUNGEN}

Nach einer Bestrahlung mit letalen Dosen treten zwei kritische Phasen auf. Ein Teil der mit mittleren Dosen und der größte Teil der mit hohen Dosen bestrahlten Tiere stirbt bereits in der ersten kritischen Phase. Tiere, die sich in dieser Phase häuten, haben eine geringe Lebenserwartung. Die zweite kritische Phase erreichen die mit niedrigen Dosen bestrahlten Tiere und die Tiere, die nach einer Bestrahlung mit mittleren Dosen die erste kritische Phase überlebt haben. Was eine niedrige, mittlere und hohe Dosis genannt werden kann, hängt von der Strahlenempfindlichkeit der Versuchstiere $a b$.

Der Zeitpunkt des Sterbens in den kritischen Phasen wird bestimmt durch den Zeitpunkt der Häutung, und zwar derart, daß in der ersten kritischen Phase der Tod in einer bestimmten Zeitspanne vor einer zu erwartenden Häutung eintritt, während er in der zweiten kritischen Periode in einer bestimmten Zeitspanne nach einer erfolgten Häutung eintritt. Eine Abhängigkeit des Zeitpunktes des Todes vom Zeitpunkt der Häutung in der zweiten kritischen Phase kann nur dann in Erscheinung treten, wenn die Zeitspanne zwischen der letzten Häutung vor dem Tode und der Phase der akuten Sterblichkeit nicht zu gering ist.

Bisherige Untersuchungen an Krebsen von ReEs (1962) und ENGEL (1967) haben $\mathrm{LD}_{50 / 30^{-}}$-Werte $z$ wischen 1500 und $51000 \mathrm{R}$ ergeben. Bonham \& Palumbo (1951) geben die $\mathrm{LD}_{50 / 20}$ für Amphipoden mit $16000 \mathrm{R}$ an. Von allen genannten Autoren wurden zur Ermittlung der Letaldosen die Schätzverfahren von ReEd \& MuENCH (1938) oder IR WIN \& ChEESEMAN (1939) benutzt, die nur dann die gesuchte „50\%ig wirksame Dosis" liefern, wenn die individuell wirksamen Dosen eine symmetrische Verteilung besitzen, eine Voraussetzung, deren Vorliegen - soweit ersichtlich - von den genannten Autoren nicht geprüft worden ist. Da Bontam \& Palumbo (1951) und Engel (1967) ihre Versuche außerdem mit relativ wenigen Tieren durchgeführt haben, können ihre Werte nur grobe Anhaltspunkte liefern. Andererseits ist bekannt, daß die Reaktionen der Organismen auf ionisierende Strahlung von zahlreichen Faktoren, wie Energie und Art der Strahlung, Dosisleistung und Stoffwechselaktivität abhängen, so daß - wie REES (1962) betont - von verschiedenen Autoren gewonnene Ergebnisse selten streng vergleichbar sind. Es mag daher in den Fällen, in denen nur angegeben werden soll, in welchem ungefähren Bereich die tödlichen Dosen zu suchen sind, die Verwendung nur weniger Versuchstiere und die Anwendung der genannten Schätzverfahren ausreichend sein.

\section{ZUSAMMENFASSUNG}

1. Der Einfluß einmaliger Röntgenbestrahlungen auf die Überlebenszeiten der euryhalinen Amphipoden Gammarus duebeni LiLujeborg, Gammarus salinus Spooner und Gammarus zaddachi SEXTON wurde bei einer konstanten. Temperatur von $15^{\circ} \mathrm{C}$ und einem Salzgehalt von $10 \%$ untersucht.

2. Bestrahlungen mit Dosen von 625 und $1250 \mathrm{R}$ führen bei $G$. duebeni zum vorzeitigen Tod von nur einigen Versuchstieren, Bestrahlungen mit $2500 \mathrm{R}$ und höheren Dosen zum vorzeitigen Tod aller Versuchstiere. 
3. Bei $G$. duebeni sind die $q Q$ strahlenempfindlicher als die $\partial \hat{\partial}$, die Jungtiere strahlenempfindlicher als adulte Individuen.

4. G. salinus und $G$. zaddachi sind strahlenempfindlicher als $G$. duebeni.

5. Die $\mathrm{LD}_{50}$-Kurven lassen drei Bereiche erkennen. Bei mittleren Dosen ändert sich die Zeitspanne, in der 50\% der Versuchstiere sterben, nur wenig oder gar nicht mit der Dosis. Bei hohen und niedrigen Dosen ist die Uberlebenszeit dosisabhängig.

6. Die Strahlenempfindlichkeit von G. duebeni war im ersten Quartal des Jahres 1967, in dem die Temperaturen uber den langfristigen Monatsmitteln lagen, geringer als bei Flohkrebsen, die zu einem späteren Zeitpunkt gefangen wurden oder aus Laboratoriumszuchten stammten.

7. Die Häutungsvorgänge, deren Beeinflussung nur bei $G$. duebeni untersucht wurde, werden durch Bestrahlungen mit $1250 \mathrm{R}$ oder geringeren Dosen nicht beeinflußt.

8. Nach einer Bestrahlung mit $20000 \mathrm{R}$ sind 2 Tage nach der Bestrahlung nur wenige oder gar keine Häutungen möglid. Nach Bestrahlungen mit 10000 und 5000 R erfolgen die Häutungen verspätet.

9. Als Folge von Bestrahlungen mit letalen Dosen nimmt die Zahl der Häutungen um den 30. Tag nach der Bestrahlung ab.

10. Die Häutungsvorgänge waren bei Tieren, die im ersten Quartal 1967 gefangen und bestrahlt wurden, weniger beeinflußbar als bei Tieren, die aus Laboratoriumszuchten stammten oder zu einem späteren Zeitpunkt gefangen wurden.

11. Nach Bestrahlungen mit letalen Dosen treten zwei kritische Phasen auf. Ein Teil der mit mittleren und der größte Teil der mit hohen Dosen bestrahlten Tiere stirbt bereits in der ersten kritischen Phase. Die zweite kritische Phase wird von allen mit niedrigen Dosen bestrahlten Tieren erreicht. Tiere, die sich in der ersten kritischen Phase häuten, haben eine geringe Lebenserwartung.

12. Die Überlebenszeit des einzelnen Individuums hängt außer von der Bestrahlungsdosis von dem Zeitpunkt ab, zu dem es sich innerhalb der Versuchszeit häutet.

Danksagung. Fräulein M. Deecke, Fräulein M. Schramm und Frau H. Rade danke ich für tednische Assistenz und die Bereitschaft, die Kontrolle der Versuche auch an arbeitsfreien Tagen zu übernehmen.

\section{ZITIERTE LITERATUR}

ANGELOViC, J. W. \& ENGEL, D. W., 1968. Interaction of gamma irradiation and salinity on respiration of brine shrimp (Arternia salina) nauplii. Radiat. Res. 35, 102-108.

- White, J. C. \& ENGEL, D. W., 1966. Influence of salinity on the response of estuarine animals to ionizing radiation. Ass. SE Biol. Bull. (= ASB Bull.) 13, 29.

Balesdent, M.-L., 1965. Recherches sur la sexualité et le déterminisme des caractères sexuels d'Asellus aquaticus Linné (Crustacé, Isopode). Bull. Acad. Soc. Lorraines Sci. 5, 1-231.

Ballardin, E. \& Metaliı, P., 1965a. Effetto dei raggi X sulla durata della vita di vari ceppi di Artemia salina LeACH. Boll. Zool. 32, 587-602.

- - 1965b. Sulla relazione fra poliploidia e radiosensibilità in ovociti di Artemia salina LEACH. Boll. Zool. 32, 613-618. 
Bonham, K. \& PAlumbo, R. F, 1951. Effects of X-rays on snails, crustacea, and algae. Growth 15, 155-188.

BoweN, S. T., 1963. The genetics of Artemia salina. III. Effects of X-irradiation and of freezing upon cysts. Biol. Bull. mar. biol. Lab., Woods Hole 125, 431-440.

BulNheIm, H.-P., 1965. Untersuchungen über Intersexualität bei Gammarus duebeni (Crustacea, Amphipoda). Helgoländer wiss. Meeresunters. 12, 349-394.

Cavaldi-Sforza, L., 1964. Grundbegriffe der Biometrie... A. d. Ital. Bearb. von R. J. LoRENZ. G. Fischer, Stuttgart, 209 pp.

Cervini, A. \& Gravelli, S., 1965. Radiosensitivity of different meiotic stages of oocytes in parthenogenetic diploid Artemia salina LEAcH. Mutation Res. 2, 452-456.

CoRK, J.M., 1957. Gamma-radiation and longevity of the flour beetle. Radiat. Res. 7, 551-557.

EASTER, S. S. \& FUTCHINSON, F., 1961. Effects of radiations of different LET on Artemia eggs. Radiat. Res. 15, 333-340.

ENGEL, D. W., 1967. Effect of single and continuous exposures of gamma radiation on the survival and grow th of the blue crab, Callinectes sapidus. Radiat. Res. 32, 685-691.

- Angelovic, J. W. \& DAvis, E. M., 1966. Effects of cobalt 60 gamma rays on the respiratory metabolism of Artemia salina nauplii and adults. A. Rep. radiobiol. Lab. Bur. comml Fish. (= Circ. Fish Wildl. Serv., Wash.) $1965(=244), 38-39$.

- \& Davis, E. M., 1965. Effects of radiation on brine shrimp. A. Rep. radiobiol. Lab. Bur. comml Fish. (= Circ. Fish Wildl. Serv., Wash.) $1964(=217), 28$.

- \& FlUKE, D. J., 1962. The effect of water content and postirradiation storage on radiation sensitivity of brine shrimp cysts (eggs). Radiat. Res. 16, 173-181.

GajewsmaJA, N., 1923. Der Einfluß der Röntgenstrablen auf Artemia salina. Verh. int. Verein. theor, angew. Limnol. 1, 359-362.

Grosch, D. S., 1962. The survival of Artemia populations in radioactive sea water. Biol. Bull. mar, biol. Lab, Woods Hole 123, 302-316.

- \& Erdman, H. E, 1955. X-ray effects on adult Artemia. Biol. Bull. mar. biol. Lab., Woods Hole 108, 277-282.

-_ \& Sullivan, R. L., 1955. X-ray induced cessation of gamete production by adult female Artemia. Biol. Bull. mar. biol. Lab., Woods Hole 109, 359 (Abstr.).

HAemmerli-Boveri, V, 1926. Über die Determination der sekundären Geschlechtsmerkmale (Brutsackbildung) der weiblichen Wasserassel durch das Ovar. Z. vergl. Pbysiol. 4, 668-698.

Hagen, C. W. \& Simmons, E. L., 1947. Effects of total body X-irradiation on rats. Pt I. Lethal action of single, paired, and periodic doses. Rep. Congr. atom. Energy Commn U.S. MDDC-1210, $1-37$.

Hutchinson, F. \& EASTER, S. S., 1960. A difference between biological effects of gamma rays and heary ions. Science, N.Y. 132, 1311-1312.

IRwIN, J.O. \& CHeEsemaN, E. A., 1939. On an approximate method of determining the median effective dose and its error, in the case of a quantal response. J. Hyg., Camb. 39, 574-580.

IWASAKI, T., 1958. The effects of water content on X-ray sensitivity in Artemia eggs. Radiat. Res. 9, 133 (Abstr. 125).

- 1959. Effects of the moisture content on $\gamma$-ray sensitivity in Artemia eggs. Bull. Inst. chem. Res. Kyoto Univ. 37, 400-404.

- 1965. Sensitivity of Artemia eggs to $\gamma$-irradiation. VII. Relationship between the degree of biological damage and the decay of free radicals in irradiated eggs. Int. I. Radiat. Biol. 9, $573-580$.

- 1966. Sensitivity of Artemia eggs to $\gamma$-irradiation. VIII. Modification of amount of radiation-induced free radicals by post-irradiation treatments. Int. I. Radiat. Biol. 11, 153-159.

KrnNe, O., 1953a, Uber den Einfluß der Temperatur auf die Geschlechrsbestimmung bei Gammarus salinus Spooner (Amphipoda). Zool. Anz. 151, 277-281.

- 1953b. Zur Biologie und Physiologie von Gammarus duebeni Lulu. 2. Uber die Häutungsfreuqenz, ihre Abhängigkeit yon Temperatur und Salzgehalt sowie uber ihr Verhalten bei isoliert gehaltenen und amputierten Versuchstieren. Zool. Jb. (Abt. allg. Zool. Pbysiol. Tiere) 64, 183-206. 
KüHL, H., 1965-1968. Wassertemperatur, Salzgehalt und andere chemische Faktoren an der "Alten Liebe" in Cuxhaven. Veröff. Inst. Küst.- u. Binnenfisch. 34, 1-19; 38, 1-19; 41, $1-19 ; 42,1-17$.

Le Rovx, M.-L., 1933. Recherches sur la sexualité des gammariens. Bull. biol. Fr. Belg. 16 (Suppl.), 1-138.

LuCA, V. DE, 1962a. Prime osservazioni sull'azione a distanza dei raggi $\mathrm{X}$ in Porcellio laevis LaTr. (Crust. Isop.). Boll. Sed. Accad. gioenia Sci. nat. 7, 135-140.

- 1962b. Sulla struttura della ghiandola meso-intestinale di Porcellio laevis Latremle (Crust. Isop.) e sue modificazioni per effetto dell'azione diretta ed a distanza dei raggi Roentgen. Archo zool. ital. 47, 225-248.

Marshall, J. S., 1962. The effects of continuous gamma radiation on the intrinsic rate of natural increase of Daphnia pulex. Ecology 43, 598-607.

- 1963. The effects of continuous, sub-lethal gamma radiation on the intrinsic rate of natural increase and other population attributes of Daphnia pulex. In: Radioecology. Ed. by V. Schultz \& A. W. Klement. Reinhold, New York, 363-366.

- 1966. Population dynamics of Daphnia pulex as modified by chronic radiation stress. Ecology 47, 561-571.

- 1967. Radiation stress in exploited Dapbnia populations. Limnol. Oceanogr. 12, 154-158.

Metalli, P. \& Ballardin, E., 1962. First results on X-ray-induced genetic damage in Artemia salina LeACH. Atti Ass. genet. ital. 7, 219-231.

- - 1963. Radiobiological problems in Artemia salina. Int. J. Radiat. Biol. 6, 376 (Abstr.).

Morgan, F., 1960. Fisheries radiobiology and the discharge of radioactive wastes. In: Disposal of radioactive wastes. International Atomic Energy Agency (IAEA) Vienna 2, 17-24.

Morr, Y., 1933a. Kastrationsversuche bei Cladoceren. I. Die Entwicklung der sekundären Sexualcharaktere bei radiumbestrahlten Männchen von Daphnia magna. Z. wiss. Zool. 144, 289-316.

- 1933b. Kastrationsversuche bei Cladoceren. II. Die Entwidklung der sekundären Sexualcharaktere bei radiumbestrahlten Weibchen von Daphnia magna. Z. wiss. Zool. 144, 573-612.

Patanè, L. \& LuCa, V. DE, 1956. Azione dei raggi X sullo sviluppo degli oostegiti di Porcellio laevis Latreille (Crust. Isopoda). Boll. Soc. Biol. sper. 32, 1512-1515.

Rajewsky, B. \& Schraub, A., 1948. Allgemeine Schädigungskurven. In: Naturforschung und Medizin in Deutschland 1939-1946. Bd 21. Biophysik. T. 1. Hrsg. von B. RAJEWSKY \& M. SснӧN. Dietrich, Wiesbaden, 123-131.

Reed, L. J. \& Muench, H., 1938. A simple method of estimating fifty per cent endpoints. Am. J. Hyg. 27, 493-497.

ReEs, G. H., 1962. Effects of gamma radiation on two decapod crustaceans, Palaemonetes pugio and Uca pugnax. Chesapeake Sci. 3, 29-34.

RICE, T. R., 1965. Long-term effects of cesium 137 on a copepod. A. Rep. radiobiol. Lab. Bur. comml Fish. (= Circ. Fish Wildl. Serv., Wash.) $1963(=204), 10$.

SAchs, L., 1968. Statistische Auswertungsmethoden. Springer, Berlin, 671 pp.

Schütz, L., 1964. Die tierische Besiedlung der Hartböden in der Schwentinemündung. Kieler Meeresforsch. 20, 198-217.

- \& KINNE, O., 1955. Uber die Mikro- und Makrofauna der Holzpfähle des Nord-OstseeKanals und der Kieler Förde. Kieler Meeresforsch. 11, 110-135.

SNider, G. \& Kersten, H., 1935. The action of soft X-rays on cladocera (Daphnia magna). Pbysiol. Zoöl. 8, 530-538.

- - 1936. Susceptibility to soft X-rays of Daphnia magna during its development from egg to young in the brood pouch. J. exp. Zoöl. 74, 1-6.

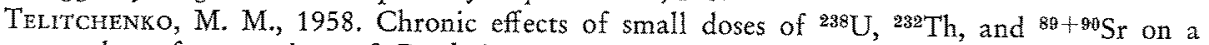
number of generations of Daphnia magna Straus. [Russ.] Nauch. Dokl. wȳssh. Shk. 1, $114-118$.

Vitagliano-Tadini, G. \& CAPOA, A. DE, 1962. Dati preliminari sull'azione dei raggi X sugli embrioni di Asellus aquaticus L. (Crost. Isop.). Atti Soc, pelorit. Sci. fis. mat. nat. $\mathbf{8}_{3}$ $133-143$. 
White, J. C. \& Angelovic, J. W., 1966. Tolerances of several marine species to Co 60 irradiation. Chesapeake Sci. 7, 36-39.

- - Engel, J. W. \& Davis, E. M., 1967. Interactions of radiation, salinity and temperature on estuarine organisms. A. Rep. radiobiol. Lab. Bur. comml Fish. $(=$ Circ. Fish Wildl. Serv., Wash.) $1966(=270), 29-35$.

Anschrift des Autors: Dr. M. Hoppenhert

Biologische Anstalt Helgoland

Zentrale

2 Hamburg 50

Palmaille 9 